\title{
Prolonged Culturing of IPSC-derived Brain Endothelial-like Cells Is Associated With Quiescence, Downregulation of Glycolysis, and Resistance to Disruption by an Alzheimer's Brain Milieu
}

\section{Lindsey M Williams}

VA Puget Sound Health Care System Seattle Division: VA Puget Sound Health Care System

\section{Takashi Fujimoto}

Nagasaki University Graduate School of Biomedical Sciences

\section{Riley R Weaver}

VA Puget Sound Health Care System Seattle Division: VA Puget Sound Health Care System

Aric F Logsdon

VA Puget Sound Health Care System Seattle Division: VA Puget Sound Health Care System

Miranda L Wilson

VA Puget Sound Health Care System Seattle Division: VA Puget Sound Health Care System

Kira M Evitts

University of Washington Department of Bioengineering

Jessica E Young

University of Washington Department of Laboratory Medicine and Pathology

\section{William A Banks}

VA Puget Sound Health Care System Seattle Division: VA Puget Sound Health Care System Michelle A Erickson ( $\square$ mericks9@uw.edu )

VA Puget Sound Health Care System Seattle Division: VA Puget Sound Health Care System https://orcid.org/0000-0002-2575-1594

\section{Research Article}

Keywords: Blood-brain barrier (BBB), human induced pluripotent stem cells (iPSCs), quiescence, glycolysis, glucose transporter-1 (GLUT1), tight junction protein (TJP), Alzheimer's disease (AD)

Posted Date: October 21st, 2021

DOI: https://doi.org/10.21203/rs.3.rs-968596/v1 
License: (c) (i) This work is licensed under a Creative Commons Attribution 4.0 International License. Read Full License

Version of Record: A version of this preprint was published at Fluids and Barriers of the CNS on February 5th, 2022. See the published version at https://doi.org/10.1186/s12987-022-00307-1. 


\section{Abstract}

Background: Human induced pluripotent stem cell (hiPSC)-derived brain endothelial-like cells (iBECs) are a robust, scalable, and translatable model of the human blood-brain barrier (BBB). Prior works have shown that high transendothelial electrical resistance (TEER) persists in iBECs for at least two weeks, emphasizing the utility of the model for longer term studies. However, most studies evaluate iBECs within the first few days of subculture, and little is known about their proliferative state, which could influence their functions. In this study, we characterized iBEC proliferative state in relation to key BBB properties at early ( 2 days) and late (9 days) post-subculture time points.

Methods: hiPSCs were differentiated into iBECs using fully defined, serum-free medium. The proportion of proliferating cells was determined by BrdU assays. We evaluated TEER, expression of glycolysis enzymes and tight and adherens junction proteins (TJP and AJP), and glucose transporter-1 (GLUT1) function by immunoblotting, immunofluorescence, and quantifying radiolabeled tracer permeabilities. We also compared barrier disruption in response to TNF- $a$ and conditioned medium (CM) from hiPSC-derived neurons harboring the Alzheimer's disease (AD)-causing Swedish mutation (APPSwe/+$)$.

Results: A significant decline in iBEC proliferation over time in culture was accompanied by adoption of a more quiescent endothelial metabolic state, indicated by downregulation of glycolysis-related proteins and upregulation GLUT1. Interestingly, upregulation of GLUT1 was associated with reduced glucose transport rates in more quiescent iBECs. We also found significant decreases in claudin-5 (CLDN5) and vascular endothelial-cadherin (VE-Cad) and a trend toward a decrease in platelet endothelial cell adhesion molecule-1 (PECAM-1), whereas zona occludens-1 (ZO-1) increased and occludin (OCLN) remained unchanged. Despite differences in TJP and AJP expression, there was no difference in mean TEER on day 2 vs. day 9. TNF-a induced disruption irrespective of iBEC proliferative state. Conversely, APPSwe/+ $\mathrm{CM}$ disrupted only proliferating iBEC monolayers.

Conclusion: iBECs can be used to study responses to disease-relevant stimuli in proliferating vs. more quiescent endothelial cell states, which may provide insight into BBB vulnerabilities in contexts of development, brain injury, and neurodegenerative disease.

\section{Introduction}

The vascular blood-brain barrier (BBB) is a vital interface that limits the unregulated transfer of circulating substances into the brain and facilitates the regulated transport of substances such as ions, nutrients, and signaling molecules that are essential for CNS homeostasis [1]. The BBB is primarily comprised of highly specialized brain endothelial cells (BECs) that confer both barrier and interface functions. The barrier functions are attributed, in part, to tight and adherens junction proteins (TJP and AJP), which effectively seal the space between neighboring BECs to prevent paracellular diffusion [2]. Suppression of transcellular leakage via pinocytic vesicles, expression of efflux transporters, and enzymatic degradation of blood-derived substances also contribute to the barrier properties of BECs $[3,4]$. 
The interface functions of the BBB include transporter-mediated passage of nutritive and regulatory substances, secretory functions, and the ability of BECs to modulate their activities in response to signals from the blood or brain compartments. Each of these functions can facilitate communication between the brain and the periphery, thus regulating CNS activities [5].

There is mounting interest in understanding the physiology of the BBB and its pathological changes in disease contexts. The development of in vitro models of the human BBB that derive BEC-like cells (iBECs) from human induced pluripotent stem cells (hiPSCs) offers a robust platform for studying many aspects of BBB function, including interactions between BECs and other cell types of the neurovascular unit (pericytes, astrocytes, neurons) [6], genetic contributions to BBB dysfunction, and mechanisms of BBB dysfunction in neurological diseases [7-11]. iBECs express TJPs and AJPs, and functional nutrient and efflux transport systems [12-15]. One major benefit of iBECs is that they develop high trans-endothelial electrical resistance (TEER) and low permeability to inert tracers, approximating in vivo barrier properties. This allows for the study of BBB transport systems with minimal confounds of leakage. In most studies, iBECs have been characterized and tested two days after subculturing the differentiated cells (postsubculture), though it has been shown that they retain strong barrier properties for at least two weeks [14, 15]. The preservation of high TEER in iBECs suggests that they can be used for longer in vitro studies. However, further characterization of the expressional and functional properties of iBECs at extended postsubculture timepoints is needed.

In this study, we characterized the phenotypic changes that occur over time in iBECs derived from the GM25256 hiPSC line by comparing protein expression and functional outcome measures at day 2 and day 9 post-subculture. These time points were selected to reflect a time conventionally used in the literature, day 2 post-subculture, and a later time to which GM25256-derived iBECs reliably sustained high TEER, day 9 post-subculture. We first noted that the amount of cellular proliferation was significantly lower in iBECs subcultured for nine days vs. two days. Subsequently, we characterized how key aspects of the BBB phenotype are altered in iBECs' more quiescent state. We focused on: 1) metabolic markers of endothelial quiescence, because an altered relation between glucose metabolism and transendothelial glucose transport may be important to consider when using iBECs to study dysfunctional GLUT1mediated glucose transport at the BBB in Alzheimer's disease (AD) [16-18], 2) markers of BBB integrity, such as TEER and expression of TJP and AJP, because BEC proliferation has been associated with BBB leakiness $[19,20]$, and 3 ) responses to inflammatory and $A D$-associated insults, which are insults that can happen concurrently with increased BEC proliferation [21, 22]. Our findings indicate that length of subculture influences the baseline phenotypes of iBECs, as well as their responses to conditioned medium (CM) from hiPSC-neurons harboring the familial AD-causing Swedish mutation. These findings provide new insight into the relations between BEC proliferative state and BBB functions at baseline and in response to disease-related insults.

\section{Methods}


iBECs were derived using the method of Neal et al [15]. Human iPSCs (hiPSCs) from the GM25256 line (Coriell Institute) were maintained on plates coated with Matrigel (Corning, cat no. 356230) in E8 Flex medium (Thermo Fisher Scientific, cat no. A28585-01). The day before differentiation was initiated, hiPSCs were dissociated into single cells with Accutase (Thermo Fisher Scientific, cat no. A1110501) and plated onto Matrigel-coated plates at a density of $15 \times 10^{3}$ cells/well in E8 Flex medium supplemented with $10 \mu \mathrm{M}$ Rho-associated protein kinase (ROCK) inhibitor Y-27632 (R\&D Systems, cat no. 1254). To initiate differentiation, the medium was changed to E6 (Thermo Fisher Scientific, cat no. A1516401) and E6 medium changes continued daily for 3 more days. Then, the medium was changed to human endothelial serum-free medium (HESFM, Thermo Fisher Scientific, cat no. 11111044) supplemented with $20 \mathrm{ng} / \mathrm{mL}$ basic fibroblast growth factor (bFGF; Peprotech, cat no. 100-18B), $10 \mu \mathrm{M}$ retinoic acid (RA; Sigma, cat no. R2625), and 1\% B27 supplement (Thermo Fisher Scientific, cat no. 17504044). After two days, iBECs were dissociated with Accutase and subcultured onto 24-well transwell permeable inserts (Corning, cat no. 3470) or tissue culture plates (Corning, cat no. 3513,3548) coated with $1 \mathrm{mg} / \mathrm{mL}$ Collagen IV (Sigma, cat no. C5533) and $5 \mathrm{mM}$ Fibronectin (Sigma, cat no. F1141) in HESFM $+20 \mathrm{ng} / \mathrm{mL}$ bFGF, $10 \mu \mathrm{M} \mathrm{RA}$, and 1\% B27 (day 0). $24 \mathrm{~h}$ later, the medium was changed to HESFM $+1 \%$ B27 without bFGF or RA, and resistance () values for monolayers of iBECs seeded on transwells were obtained for day 1 using an EVOM2 Voltohmmeter (World Precision Instruments, Sarasota Florida) coupled to an ENDOHM cup chamber. Transendothelial electrical resistance (TEER) was calculated by subtracting the resistance () value of a blank transwell and multiplying by the transwell surface area $\left(0.33 \mathrm{~cm}^{2}\right)$. TEER measurements occurred daily to confirm the integrity of the monolayers. Experiments were only conducted on iBECs with TEER $>1000{ }^{*} \mathrm{~cm}^{2}$.

\section{Immunofluorescence Analyses}

iBECs plated on 48-well plates were washed once with PBS (Thermo Fisher Scientific, cat no. 70011044) and fixed in a 1:1 methanol/acetone mixture for $15 \mathrm{~min}$ at $4^{\circ} \mathrm{C}$. Wells were washed with PBS $3 x$ for $5 \mathrm{~min}$ each, then blocked with 5\% normal donkey serum (Jackson ImmumoResearch, cat no. 017-000-121) + $0.1 \%$ TX-100 (Sigma, cat no. X100) in PBS for $1 \mathrm{~h}$ at RT. Wells were washed $3 \mathrm{x}$ for 5 min each, then iBECs were incubated with primary antibody solutions (see Table 1 for dilutions) in phosphate-buffered normal antibody diluent (NAD) (Scytek, cat no. ABB500) overnight at $4^{\circ} \mathrm{C}$. Wells were washed $3 x$ for 5 min each, then incubated with secondary antibody solutions (all 1:200) and DAPI (Thermo Fisher Scientific, cat no. 62248) (1:5000) in NAD for $1 \mathrm{~h}$ at RT. Wells were washed $3 x$ for 5 min each, then imaged using a Zeiss Axiovert 7. For each differentiation, 3 wells were designated per group and 3-4 images were taken per well. Mean fluorescence intensity (MFI) values were quantified using the Zen image analysis software and were corrected for differences in cell density via normalization to DAPI MFI.

\section{BrdU Proliferation Assays}

Analysis of 5-bromo-2'-deoxyuridine (BrdU) (Thermo Fisher Scientific, cat no. B23151) incorporation into the DNA was used to evaluate iBEC proliferation. For iBECs fixed on day 2, $10 \mu \mathrm{M}$ BrdU was included with the medium change to HESFM $+1 \%$ B27 without bFGF or RA on day 1 . For iBECs fixed on day $4,5,7$, or 9 , 
BrdU was added to a final concentration of $10 \mu \mathrm{M}$ by diluting a $100 \mu \mathrm{M}$ BrdU solution into the existing medium on day $3,4,6$, or 8 , respectively. $24 \mathrm{~h}$ after addition of $10 \mu \mathrm{M} \mathrm{BrdU}$, cells were washed $1 \mathrm{x}$ quickly with PBS and fixed in ice-cold 1:1 methanol/acetone fixative for $15 \mathrm{~min}$. at $4^{\circ} \mathrm{C}$. Cells were permeabilized with $0.1 \%$ TX-100 in PBS for 20 min at RT. DNA strands were denatured with $2 \mathrm{~N} \mathrm{HCl}$ for 10 min at RT. Then, the IFA for was performed according to the procedure outlined above.

\section{Protein Extractions and Immunoblots}

iBECs plated on 12-well plates were washed in ice-cold PBS and scraped in a RIPA-like buffer (1\% cytoplasmic stock, $1 \%$ NP-40, 0.5\% Deoxycholate, $0.1 \%$ SDS) supplemented with protease (Sigma, cat no. P8340) and phosphatase (Sigma, cat no. P5726) inhibitors. Lysates were frozen at $-80^{\circ} \mathrm{C}$, then thawed on ice and centrifuged at $20000 \mathrm{xg}$ for $5 \mathrm{~min}$ at $4^{\circ} \mathrm{C}$. The supernatant was saved and protein concentrations for each cell lysate were determined by Bradford assay (Thermo Fisher Scientific, cat no. 23200) using known concentrations of bovine serum albumin to create the protein standard curve. Samples were prepared for electrophoresis by mixing with 4X LDS Sample Buffer (Novex, cat no. NP007), 10X Sample Reducing Agent (Novex, cat no. NP0009), and molecular biology-grade water, then denatured at $70^{\circ} \mathrm{C}$ for $10 \mathrm{~min}$. Protein mixtures containing $10 \mathrm{mg}$ of protein were electrophoresed using ExpressPlus PAGE precast gels (GeneScript, cat no. M41210), then electrotransferred to nitrocellulose membranes (Invitrogen, cat no. IB301002) using the iBlot Dry Blotting System (Invitrogen). Nonspecific binding was blocked by incubating the membrane in Tris-buffered saline $0.1 \%$ Tween-20 (TBS-T) supplemented with 5\% BSA (Sigma, cat no. A7030) at RT for 45 min. Primary antibody solutions (see Table 1 for dilutions) were prepared in $5 \%$ BSA/TBS-T and membrane incubations occurred overnight at $4^{\circ} \mathrm{C}$. Membranes were washed with TBS-T three times for 5 min each, then incubated with horseradish peroxidase conjugated secondary antibodies (all 1:5000) at RT for $30 \mathrm{~min}$. Then, membranes were washed with TBS-T three times for 5 min each followed by one TBS wash for $1 \mathrm{~min}$. West Pico chemiluminescence reagent (Thermo Fisher Scientific, cat no. PI-34078) was applied, and bands of immunoreactive protein were visualized with the ImageQuant LAS4000 (Cytiva Life Sciences, formerly GE Healthcare Life Sciences) Western blot Imaging system or the Amersham ${ }^{\text {TM }}$ ImageQuant 800 (Cytiva Life Sciences, formerly GE Healthcare Life Sciences). Quantification of the band volumes was performed with ImageQuant TL 1D Gel Analysis Software (Cytiva Life Sciences, formerly GE Healthcare Life Sciences).

\section{Glucose Transport Assays}

Transwells were distributed such that the mean TEER values were approximately equal among groups. The luminal and abluminal HESFM $+1 \%$ B27 mediums were refreshed, and cells were equilibrated in the incubator for $20 \mathrm{~min}$. The input medium consisted of HESFM + 1\% B27 containing $0.1 \mu \mathrm{Ci}{ }^{14} \mathrm{C}-2$ deoxy-Dglucose ( ${ }^{14} \mathrm{C}-\mathrm{DG}$, FW $164.16 \mathrm{~g} / \mathrm{mol}$ ) (Perkin Elmer) and 1 million CPM ${ }^{99 \mathrm{~m}} \mathrm{Tecnicium}$

diethylenetriaminepentaacetic acid ( ${ }^{99 \mathrm{~m}}$ Tc-DTPA, FW $487.21 \mathrm{~g} / \mathrm{mol}$ ) per transwell. As 2-deoxy-D-glucose is transported at nearly the same rate as glucose and is trapped intracellularly after hexokinase phosphorylation, it is an appropriate tracer to measure transcellular glucose transport [23]. The Pe for ${ }^{99 m}$ Tc-DTPA was used to measure non-specific para- and transcellular leakage. To evaluate the saturability of the iBEC glucose transport system, the input medium was supplemented with $D(+)-$ 
Glucose (Sigma, cat no. G7528) or D-Mannitol (Sigma, cat no. M4125). BAY-876 (Tocris, cat no. 6199) was obtained as a powder and stored at $-20^{\circ} \mathrm{C}$ in a glass vial. Stock solutions ( $\left.2 \mathrm{mM}\right)$ were prepared by diluting BAY-876 in DMSO (Sigma, cat no. D2650) (vehicle control) and were stored in $25 \mu \mathrm{L}$ aliquots at $-70^{\circ} \mathrm{C}$. The input medium was supplemented BAY-876 to a final concentration of $2 \mu \mathrm{M}$ to evaluate GLUT1 inhibition. For assays evaluating luminal-to-abluminal (blood-to-brain) transport, the $100 \mu \mathrm{L}$ luminal (donor) chamber volume was switched to the input medium to initiate the assay, and $500 \mu \mathrm{L}$ volumes of medium from the abluminal (receptor) chamber were collected and replaced with fresh, pre-warmed HESFM $+1 \%$ B27 medium after incubation times of 5, 10, 15, and $20 \mathrm{~min}$. Of the $500 \mu \mathrm{L}$ total volume collected, $200 \mu \mathrm{L}$ was added to liquid scintillation vials containing $5 \mathrm{~mL}$ of Ecoscint ${ }^{\text {TM }}$ (National Diagnostics). The vials were dark-adapted for at least 4 days. Then, the radioactivity was counted in a Tricarb beta counter (Perkin Elmer) to measure ${ }^{14} \mathrm{C}-\mathrm{DG}$ levels. The radioactivity in the remaining $300 \mu \mathrm{L}$ was counted in a Wizard 2 gamma counter (Perkin Elmer) to measure ${ }^{99 m}$ Tc-DTPA levels. For abluminalto-luminal (brain-to-blood) transport assays, the $600 \mu \mathrm{L}$ abluminal (donor) chamber volume was switched to an input medium to initiate the assay, and $90 \mu \mathrm{L}$ volumes of medium from the luminal (receptor) chamber were collected and replaced with fresh, pre-warmed HESFM + 1\% B27 medium after incubation times of $5,10,15$, and $20 \mathrm{~min}$. Of the $90 \mu \mathrm{L}$ collection, $40 \mu \mathrm{L}$ was counted in the beta counter and the remaining $50 \mu \mathrm{L}$ was counted in the gamma counter. Permeability-surface area coefficient $(\mathrm{Pe})$ calculations were performed according to the method of Dehouck et al., 1990 [24]. Clearance was expressed as $\mu \mathrm{L}$ of radioactive tracer transported from the donor chamber to the receptor chamber, and was calculated from the initial level of radioactivity added to the donor chamber and the final level of radioactivity in the receptor chamber:

Clearance $(\mu \mathrm{L})=[\mathrm{C}]_{\mathrm{C}} \times \mathrm{V}_{\mathrm{C}} /[\mathrm{C}]_{\mathrm{L}}$

Where $[C]_{L}$ is the initial concentration of radioactivity in the donor chamber (in units of $C P M / \mu L$ ), $[C]_{C}$ is the concentration of radioactivity in the receptor chamber (in units of $\mathrm{CPM} / \mu \mathrm{L}$ ) and $\mathrm{Vc}$ is the volume of the receptor chamber in $\mathrm{mL}$. The volume cleared was plotted vs. time, and the slope was estimated by linear regression. The slopes of clearance curves for the iBEC monolayer plus Transwell@ membrane was denoted by $\mathrm{PS}_{\mathrm{app}}$, where PS is the permeability $\times$ surface area product (in $\mu \mathrm{L} / \mathrm{min}$ ). The slope of the clearance curve for a Transwell $®$ membrane without iBECs was denoted by PS $_{\text {membrane. The PS value for }}$ the iBEC monolayer $\left(\mathrm{PS}_{\mathrm{e}}\right.$ ) was calculated from $1 / \mathrm{PS}_{\mathrm{app}}=1 / \mathrm{PS}_{\text {membrane }}+1 / \mathrm{PS}_{\mathrm{e}}$. The $\mathrm{PS}_{\mathrm{e}}$ values were divided by the surface area of the Transwell ${ }^{\circledR}$ inserts $\left(0.33 \mathrm{~cm}^{2}\right)$ to generate the endothelial permeability coefficient $\left(\mathrm{Pe}\right.$, in $\left.\mu \mathrm{L} / \mathrm{min} / \mathrm{cm}^{2}\right)$.

\section{TNF- Treatments}

Recombinant human tumor necrosis factor (TNF)-a (R\&D Systems, cat no. 210-TA) was obtained as a lyophilized powder and stored at $-20^{\circ} \mathrm{C}$ in a glass vial. Stock solutions $(100 \mu \mathrm{g} / \mathrm{mL})$ were prepared by reconstituting in sterile PBS and were stored in $25 \mu \mathrm{L}$ aliquots at $-70^{\circ} \mathrm{C}$. Stock solutions were diluted in the 
existing luminal and abluminal mediums for $100 \mathrm{ng} / \mathrm{mL}$ TNF-a treatments. After $16 \mathrm{~h}$, TEER was measured.

\section{Derivation of Neurons from hiPSCs and Conditioned Medium Treatments}

We used previously established gene-edited hiPSC lines harboring the amyloid precursor protein (APP) Swedish mutation (Swe) and as well as the isogenic control described in Young et al., 2018 [25]. This mutation corresponds to a $2 \mathrm{bp}$ change, GA to TC, in exon 16 of $A P P$. We used cell heterozygous for this mutation, APPSwe/ ${ }^{+}$, along with the isogenic APP wild-type, APP ${ }^{W T}$. Neurons from these cell lines were differentiated from hiPSCs as previously described in Knupp et al., 2020 [26] and Rose et al., 2018 [27]. hiPSC-neurons from the APPSwe/+ and APPWT lines were cultured in Neural Differentiation media (1:1 DMEM/F12 (Life Technologies, cat no. 11039047) + neurobasal media (GIBCO, cat no. 21103049), 0.5\% N2 supplement (Thermo Fisher Scientific, cat no. 17502-048) 1\% B27 supplement (Thermo Fisher Scientific, cat no. 17504-044), 0.5\% GlutaMax (Thermo Fisher Scientific, cat no. 35050061), 0.5\% insulintransferrin-selenium (Thermo Fisher Scientific, cat no. 41400045), 0.5\% NEAA (Thermo Fisher Scientific, cat no. 11140050), 0.2\% ß-mercaptoethanol (Life Technologies, cat no. 21985023), $0.2 \mu \mathrm{g} / \mathrm{mL}$ brainderived neurotrophic factor (Eurotech, cat no. 450-02), $0.2 \mu \mathrm{g} / \mathrm{mL}$ glial-cell-derived neurotrophic factor (PeproTech, cat no. 450-10), and 0.5 M dbcAMP (Sigma Aldrich, cat no. D0260) and cultured on Matrigel (Corning, cat no. 356231 ) at $37^{\circ} \mathrm{C}$ in a $5 \% \mathrm{CO}_{2}$ incubator. After 21 days of differentiation in Neural Differentiation media, conditioned medium (CM) was collected from each cell line following 72 hours of contact with the mixed neuronal cell population. $A \beta$ peptide content in $C M$ was quantified using an $A \beta$ Triplex ELISA plate (Meso Scale Discovery, cat no. 151200E-2). For iBEC conditioned medium (CM) treatments, transwells were distributed among groups (APPWT or APPSwe/+ ) such that mean TEER values were approximately equal. The abluminal medium was replaced with $\mathrm{CM}$ collected from APPWT or $\mathrm{APP}^{\mathrm{Swe} /+}$ neuronal cultures. After $24 \mathrm{~h}$, TEER was measured.

\section{Statistics}

The Prism 9.0 statistical software package was used for all statistical calculations (GraphPad Inc, San Diego, CA). Means are reported with their standard error (SE). For all figures, means are displayed with their SE and $n$. Linear regression lines and their slopes and intercepts were calculated using the Prism 9.0 software. Unpaired two-tailed t-tests were used to compare two means and analysis of variance (ANOVA) followed by Tukey's multiple comparisons test when more than two means were compared.

\section{Results}

\section{iBEC Proliferation Rate Decreases Over Time Spent in Culture}

In the adult vasculature, contact-inhibited endothelial cells (ECs) remain mostly quiescent [28]. In vitro studies using primary human ECs have shown that ECs adopt a quiescent state upon contact inhibition, which is associated with altered regulation of glucose metabolism and transport [29]. We hypothesized 
that iBECs would mature into a more quiescent state over an extended period of time in culture. To test this hypothesis, we assessed the DNA incorporation of 5-bromo-2'-deoxyuridine (BrdU) to determine the proportion of dividing cells at early ( 2 days) and late ( 9 days) post-subculture time points. We found that, over time, there was a significant decrease in the number of proliferating iBECs. (Fig. 1a-C). The percentage of BrdU positive cells was significantly lower in iBECs on day 9 post-subculture (31.63 \pm $1.59 \%)$ relative to day $2(74.80 \% \pm 2.41 \%)$, corresponding to a $57.71 \%$ reduction in proliferation over one week in standard culture conditions with no medium changes (NMC). To determine whether nutrient replenishment can restore iBEC proliferation, we tested the effects of a single medium change (SMC) on the rate of BrdU incorporation (Fig. 1b). SMC on day 8 post-subculture induced a modest increase in iBEC proliferation on day 9 compared to NMC (32.37\% $\pm 2.13 \%$ vs. $43.05 \% \pm 2.46 \%$, respectively) (Fig. $1 \mathrm{~d})$. We also tested whether re-application of the pro-angiogenic effectors basic fibroblast growth factor (bFGF) and retinoic acid (RA) on day 8 could stimulate iBEC proliferation and found no change in BrdU incorporation on day 9 in response to bFGF/RA treatment (Figure S1). Given the slight, but significant, effect of SMC on iBEC proliferation on day 9, we aimed to determine whether the decline in iBEC proliferation occurs dependently or independently of nutrient depletion in the medium over time. We performed continual medium changes (CMC) of both the luminal and abluminal mediums on days 4, 6 and 8 post-subculture, and examined the effects on BrdU incorporation (Fig. 1b). The proliferative status of iBECs on day 9 with NMC was not significantly different than with CMC (Fig. 1e). In fact, no significant differences between the NMC and CMC groups were detected on any day. Two-way ANOVA comparing the NMC vs. CMC groups on days 5,7 , and 9 revealed a significant ( $* \star * p<0.001)$ main effect of day in subculture accounting for $78.39 \%$ of the total variation, while the effects of medium changes accounted for only $0.11 \%$. While the decline in proliferation from day 2 to day 9 post-subculture is marginally reversible with nutrient replenishment on day 8 , recurring nutrient replenishment does not significantly affect iBEC proliferation, demonstrating that $\mathrm{BEEC}$ adoption of a more quiescent state is only minimally influenced by diminishing nutrient availability in the medium over time.

\section{iBEC Expression Patterns Reflect the Endothelial Transition to a More Quiescent Metabolic State}

ECs predominantly derive ATP from glucose metabolism to lactate through glycolysis, rather than oxidative phosphorylation [30]. As ECs become quiescent, they adapt to lower metabolic requirements by repressing their glycolytic activity, characterized by downregulation of 6-phosphofructo-2-kinase/fructose2,6-biphosphatase 3 (PFKFB3), which produces large amounts of fructose-2,6-bisphosphate to activate phosphofructokinase-1 (PFK1), a rate-limiting enzyme in glycolysis. Upregulation of GLUT1 is concomitant with the reduction in glycolysis, suggesting that the majority of glucose entering via GLUT1 is transported across the quiescent endothelium, while only a minor fraction is used for energy metabolism [29, 31]. Therefore, we aimed to determine whether iBECs adopt similar expression changes to primary ECs during the transition to a more quiescent state, and the effects of $\mathrm{CMC}$. We found that, with NMC, iBEC expression of PFKFB3 was significantly reduced on day 9 relative to day 2, while GLUT1 expression was significantly increased (Fig. 2a-C). PFKFB3 expression on day 9 with CMC was similar to that with NMC. However, GLUT1 expression on day 9 was significantly lower with CMC than with NMC, suggesting that GLUT1 upregulation may be, in part, enhanced by glucose depletion. Furthermore, we 
observed a greater abundance of lower molecular weight (MW) GLUT1 isoforms with NMC, while higher $\mathrm{MW}$ isoforms predominated with CMC. As glucose is a primary building block for glycosylation, this finding is consistent with predominance of more glycosylated GLUT1 isoforms when glucose is replete in the medium. We compared the GLUT1 MW profile of iBECs on day 2 and day 9 with that of primary human astrocytes. We found that iBECs on day 2 expressed higher MW isoforms than primary human astrocytes, consistent with the higher extent of glycosylation on brain endothelial GLUT1 compared to astrocytic GLUT1 [16]. In comparison, iBECs on day 9 expressed both the higher isoforms of day 2 iBECs and the lower MW isoforms of primary human astrocytes (Fig. S2). In addition, we evaluated expression changes of hexokinase 2 (HK2), which catalyzes the first rate-limiting reaction of glycolysis by phosphorylating glucose [32], and monocarboxylate transporter-1 (MCT1), which facilitates the bidirectional transport of lactate, a metabolic waste product of glycolysis and a signaling agent that promotes angiogenesis [33]. We found HK2 expression was significantly decreased in iBECs on day 9 relative to day 2, which was maintained with CMC (Fig. 2a, d). MCT1 expression was also significantly reduced in iBECs from day 2 to day 9. Interestingly, downregulation of MCT1 was enhanced by CMC (Fig. $2 \mathrm{a}, \mathrm{e})$. Overall, the glucose metabolism and transport-related protein expression changes exhibited by more quiescent iBECs reflect a systematic repression of glycolysis, recapitulating the phenotype of quiescent primary ECs.

\section{Transendothelial Glucose Transport System Function is Enhanced in Proliferating iBECs}

To determine whether the increased GLUT1 expression observed in more quiescent iBECs is accompanied by enhanced glucose transport kinetics, we assayed transendothelial transport of ${ }^{14} \mathrm{C}$-2-deoxy-D-glucose $\left({ }^{14} \mathrm{C}-\mathrm{DG}\right)$ across monolayers of iBECs on day 2 and day 9 post-subculture. As ${ }^{14} \mathrm{C}$-DG becomes trapped in the cell once it is phosphorylated by hexokinase, only unmetabolized ${ }^{14} \mathrm{C}$-DG is transported to exclusively proxy transendothelial glucose transport $[23,34]$. The permeability-surface area coefficients $(\mathrm{Pe})$ for luminal-to-abluminal and abluminal-to-luminal ${ }^{14} \mathrm{C}-\mathrm{DG}$ transport were measured in the presence and absence of excess glucose in the donor chamber (55.5 mM Glucose and $5.5 \mathrm{mM}$ Glucose, respectively) to assess glucose transport system saturability. The Pe for ${ }^{99 \mathrm{~m}}$ Tc-DTPA was measured simultaneously to measure non-specific para- and transcellular leakage. Surprisingly, we found a significant $39.33 \%$ decrease in the unsaturated rate of luminal-to-abluminal ${ }^{14} \mathrm{C}$-DG transport in iBECs on day 9 relative to day 2 (Fig. 3a). No significant effect of either day in subculture or excess glucose on iBEC permeability to $99 \mathrm{~m}$ Tc-DTPA was detected (Fig. S3a). We found a similar $36.44 \%$ decrease in the unsaturated rate of abluminal-to-luminal ${ }^{14} \mathrm{C}$-DG transport from day 2 to day 9 , evidencing a reduced capacity of the bidirectional glucose transport system in iBECs' more quiescent state (Fig. 3b). In this experiment, a significant $(* \star * p<0.001)$ main effect of day in subculture was detected where abluminal-to-luminal leakage of ${ }^{99 m}$ Tc-DTPA was significantly increased on day 9 relative to day 2 (Fig. S3b). Saturation with excess glucose in the donor compartment produced significant reductions in ${ }^{14} \mathrm{C}-\mathrm{DG}$ on both day 2 and day 9 , demonstrating the $\mathrm{iBEC}$ glucose transport system is similarly saturable in both proliferative and more quiescent states. We confirmed that the significant decrease in ${ }^{14} \mathrm{C}-\mathrm{DG}$ Pe upon the addition of excess glucose was due to saturation of the glucose transport system rather than hyperosmotic stress by 
comparing the effect of $55.5 \mathrm{mM}$ glucose vs. $55.5 \mathrm{mM}$ mannitol. $55.5 \mathrm{mM}$ mannitol did not significantly affect either ${ }^{14} \mathrm{C}$-DG Pe (Fig. S3c) or ${ }^{99 \mathrm{~m}} \mathrm{Tc}$-DTPA Pe (data not shown). In addition, we confirmed that ${ }^{14} \mathrm{C}$ DG Pe could be inhibited by the GLUT1-selective inhibitor BAY-876. $2 \mu \mathrm{M}$ BAY-876 produced significant reductions in ${ }^{14} \mathrm{C}$-DG Pe on both day 2 and day 9 post-subculture (34.96\% and $34.20 \%$, respectively) (Fig. S3d) and did not significantly affect ${ }^{99 \mathrm{~m}}$ Tc-DTPA Pe on either day (data not shown). To test whether the rate of glucose transport is regulated by nutrient availability in the medium, we compared ${ }^{14} \mathrm{C}-\mathrm{DG} P e$ on day 9 in iBECs with NMC vs. CMC. ${ }^{14} \mathrm{C}$-DG Pe was the same in both conditions (Fig. 3c), demonstrating that changes in nutrient availability likely do not mediate the difference in the glucose transport system functionality between proliferative and more quiescent iBECs. In summary, our findings indicate that 1) while the expression level of GLUT1 is regulated by changes in nutrient availability, the rate of transendothelial glucose transport is not, and 2) increased GLUT-1 expression as iBECs become more quiescent does not correspond with increased functional glucose transport.

\section{Tight and Adherens Junction Protein Expression Changes as iBEC Proliferation Declines Do Not Correlate with TEER}

Because we observed changes in the regulation of glucose metabolism and transport, we hypothesized that iBECs undergo other BBB phenotypic changes during their transition to a more quiescent state. Therefore, we next aimed to characterize changes in TJP and AJP expression in relation to TEER in proliferative vs. more quiescent iBECs. Immunofluorescence analyses (IFA) comparing TJP expression on day 2 vs. day 9 post-subculture indicated a significant decrease in claudin-5 (CLDN5) expression while occludin (OCLN) expression remained unchanged and zona occludens-1 (ZO-1) expression increased (Fig $4 a, b-d)$. Further, we detected a significant decrease in expression of the AJP vascular endothelialcadherin (VE-Cad), while there was a trend towards a decrease in platelet endothelial cell adhesion molecule-1 (PECAM-1) expression ( $p=0.0544$ ) (Fig. 4a, e-f). Immunoblot analyses of CLDN5 and OCLN expression confirmed the results of the IFA (Fig. $4 \mathrm{~g}-\mathrm{i})$. Though changes in junctional protein expression are commonly associated with changes in barrier functions, we found that TEER trajectories from day 2 to day 9 post-subculture did not show consistent patterns of change, and that there was no difference of mean TEER on day 2 vs. on day 9. (Fig. S4). The high TEER values of iBECs on day 2 suggest that the high rate of proliferation does not negatively impact BBB tightness. Conversely, the high TEER values of iBECs on day 9 suggest that the reduced expression of the key junctional proteins CLDN5 and VE-Cad is not detrimental to junctional integrity. Further, both the maintenance of high TEER and our qualitative observations of differences in junctional localization on day 2 vs. day 9 suggest that junctional remodeling occurs during the transition to a more quiescent state. It is possible that reduced proliferation allows for protein complex stabilization, requiring less intracellular trafficking and resulting in enhanced junctional localization of TJP and AJP in more quiescent iBEC monolayers. Our results show that while certain TJP and AJP levels may be influenced by iBEC proliferative state, strong barrier functions are maintained in both iBECs' proliferative and more quiescent iBEC states, supporting use of this model to study mechanisms of junctional destabilization in both states. 


\section{iBEC Proliferation Confers a Selective Vulnerability to an Alzheimer's Brain Milieu, But Not TNF- a}

We next determined whether the proliferative state of iBECs affects their vulnerability to an inflammatory stimulus. We compared the effects of $100 \mathrm{ng} / \mathrm{mL}$ TNF-a treatment (applied to both luminal and abluminal compartments) on day 2 vs. day 9 post-subculture. We found significant decreases in TEER after treatment on both days $(29.12 \%$ and $37.17 \%$, respectively), indicating that iBEC vulnerability to TNFa-induced BBB disruption is not dependent on iBEC proliferative status (Fig. 5a-d). Finally, we investigated whether an Alzheimer's disease (AD)-associated stimulus also produced comparable disruption in proliferative and more quiescent iBEC states. We modeled neuron-endothelial interactions in $A D$ by treating iBECs on the abluminal side with conditioned medium (CM) collected from neuronal cultures derived from hiPSCs harboring one copy of the Swedish mutation (APPSwe/+ ${ }^{\text {) }}$ (Fig. 6a). This familial ADcausing mutation renders the amyloid precursor protein (APP) a more favorable substrate for cleavage by $\beta$-secretase (BACE-1), causing increased production of amyloid beta (A) [35]. We compared this with CM collected from hiPSC-neurons derived from the isogenic control line, APPWT, which does not harbor the Swedish mutation. Based on previously published reports indicating enhanced susceptibility of proliferating ECs to A [36], we predicted that greater reductions in TEER would be induced by APPSwe/+-CM treatment on day 2 vs. on day 9 . On day 2 , treatment of iBECs with APPSwe/ ${ }^{+}-\mathrm{CM}$ produced a significant decrease in TEER compared with control CM from the APPWT-derived hiPSC-neurons (Fig. 6b-c). In contrast, we did not observe a significant decrease in TEER relative to Cvi-A2 control following APPSwe/-CM treatment on day 9 (Fig. $6 \mathrm{~d}-\mathrm{e}$ ). Our data suggest that the higher rate of iBEC proliferation on day 2 confers a selective vulnerability to paracellular disruption caused by an Alzheimer's brain-like milieu. Though APPSwe/+ - CM contains elevated A-40 and -42 levels compared to APPWT [25] (Fig. S5), future studies are needed to elucidate whether the effects of $\mathrm{APP}{ }^{\mathrm{Swe} /+}-\mathrm{CM}$ are A-mediated.

\section{Discussion}

A unique feature of the iBEC model is its ability to maintain high TEER over a sustained period of time in culture. The maintenance of barrier properties suggests that iBECs are amenable to longer in vitro studies, which could aid the understanding of human disease mechanisms. However, little is currently known about the stability of the iBEC phenotype with prolonged culture. As we demonstrate here, iBECs become more quiescent over time, and adopt a metabolic phenotype that is similar to primary ECs cultured to contact-inhibition [29]. We first posited that factors inherent to the culture method such as nutrient availability and/or the time elapsed from bFGF and RA exposure could potentially regulate the proliferative status of iBECs [37]. However, we found that continual medium changes (CMC) could not prevent the transition to a more quiescent state that occurs from day 2 to day 9 post-subculture, nor could the re-application of bFGF/RA fully restore the proliferative status of iBECs after prolonged culture. Only a modest increase in proliferative cells was observed when medium was changed on day 8 post-subculture. These findings indicate that the growth arrest that occurs in iBECs is stable and occurs independently of nutrient depletion or the absence of bFGF and RA. 
Prior works have characterized iBECs as primarily utilizing glycolysis vs. oxidative phosphorylation for ATP synthesis [9], which aligns with the metabolic phenotype of primary ECs [30,38]. Here, we demonstrate that iBECs undergo a metabolic transition from day 2 to day 9 post-subculture that resembles that of quiescent primary ECs, which alter their metabolism from the high energy requirements of proliferation to the lower energy requirements of quiescent barrier maintenance by downregulating glycolysis $[29,31]$. The distinctive expression changes induced in primary ECs upon contact-inhibition, namely, downregulation of PFKFB3 and upregulation of GLUT1, also occur in iBECs during the transition from a highly proliferative state on day 2 post-subculture to a more quiescent state on day 9 postsubculture. Importantly, we found that nutrient replenishment with CMC did not alter the expression of PFKFB3. Nutrient replenishment did significantly suppress the increase in GLUT1 expression, indicating that GLUT1 expression is regulated by nutrient availability in addition to the proliferative status of iBECs. This finding is consistent with increased BBB GLUT1 expression in response to hypoglycemia [39].

We further identified reduced HK2 expression and reduced MCT1 expression as two additional indicators of glycolytic repression in iBECs. HK2 is necessary to support EC proliferation as it catalyzes the first ratelimiting reaction in glycolysis, and its depletion inhibits glycolysis and impairs angiogenesis [32, 40]. Like PFKFB3, HK2 downregulation over time was not affected by CMC. MCT1 facilitates the bidirectional transport of lactate. As MCT1 expression is induced by lactate [41], decreased lactate production via glycolysis likely contributes the reduced MCT1 expression in more quiescent iBECs on day 9. Furthermore, lactate has been shown to function as a pro-angiogenic effector to promote proliferation in BECs [42]. Therefore, downregulation of MCT1 may also be a mechanism to inhibit iBEC proliferation by decreasing lactate influx. CMC further suppressed the expression of MCT1, which may be attributed to removal of the lactate produced by the iBECs during glycolysis. Overall, our findings show that more quiescent iBECs adopt a metabolic phenotype reminiscent of quiescent primary ECs. Our results highlight the utility of the model as a platform to study the regulation of BBB functions under defined proliferative and metabolic states.

We confirmed a previous report of a functional and saturable iBEC glucose transport system [42] and that a GLUT1-selective inhibitor, BAY-876, significantly inhibits glucose transport in GM25256 iBECs. Endothelial upregulation of GLUT1 that occurs concurrently with quiescence is considered to be a mechanism of prioritizing glucose delivery to the surrounding tissues by more mature cells [29]. Therefore, we predicted that more quiescent iBECs, which were shown to have higher GLUT1 protein levels, would facilitate higher rates of glucose transport. However, the rate of glucose transport, as measured by ${ }^{14} \mathrm{C}$-DG Pe, was significantly lower in more quiescent iBECs vs. proliferative iBECs, which

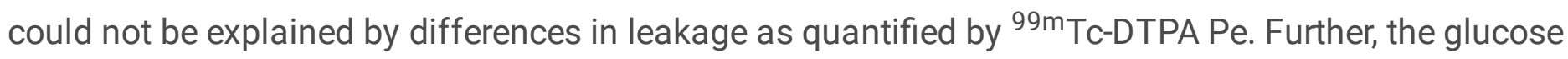
transport rate of $\mathrm{iBEC}$ on day 9 post-subculture was not significantly altered by $\mathrm{CMC}$, showing that nutrient depletion does not contribute to decreased glucose transport in more quiescent iBECs.

${ }^{14} \mathrm{C}-\mathrm{DG}$ Pe is analogous to fluorodeoxyglucose (FDG), which is an ${ }^{18} \mathrm{~F}$-labeled PET ligand that is routinely used to evaluate glucose uptake into tissues, including the brain [43]. Decreased brain uptake of FDG can be indicative of impaired glucose utilization by neurons, but also GLUT1 dysfunction at the BBB [44, 45]. 
An advantage of using ${ }^{14} \mathrm{C}-\mathrm{DG}$ as a glucose surrogate is that only unmetabolized ${ }^{14} \mathrm{C}$-DG can fully traverse the iBEC monolayer since it becomes trapped within the cell following phosphorylation to 2deoxyglucose-6-phosphate by hexokinase, and this form is unable to be further metabolized by glycolytic enzymes [23,34]. Although our assays did not have the sensitivity to detect intracellular 2-deoxyglucose6-phosphate accumulation, we posit that the reduced transport ${ }^{14} \mathrm{C}-\mathrm{DG}$ in more quiescent iBECs would not be due to increased metabolism, since glycolytic enzymes, including HK2, are reduced. Instead, the reduced transport of ${ }^{14} \mathrm{C}$-DG in more quiescent iBECs indicates that upregulation of GLUT1 protein expression does not contribute to increase glucose transport. Decreased glucose transport may be, in part, due to the localization of GLUT1 to a subcellular location other than the plasma membrane. It has been shown that GLUT1 translocation to the plasma membrane is one mode of glucose transport upregulation, which can occur without changes in GLUT1 protein expression [46]. Further, it has been shown that up to $40 \%$ of GLUT1 is sequestered in the cytoplasm of BECs [47].

In addition to observing differences in the total amount of GLUT1, we also observed the appearance of lower MW isoforms of GLUT1 in more quiescent iBECs, suggesting differences in post-translational modifications (PTM). Glycosylation is one PTM which alters the MW of GLUT1 [48], and can also regulate its function by facilitating membrane clustering on lipid rafts [49]. However, some of the apparent shifts in GLUT1 MW were mitigated by medium changes, which had no effect on functional glucose transport in our studies. It is also possible that other PTMs such as phosphorylation could regulate GLUT1 activity [50]. Presently, very little is known about the mechanisms of GLUT1 regulation in proliferating vs. quiescent BECs. iBECs could potentially be used to delineate these mechanisms as a future direction.

We and others have consistently observed the preservation of high TEER in iBECs over extended periods of time in subculture $[14,15]$. Here, we demonstrate that TEER is not dependent on length of subculture, as strong barrier functions were upheld in both proliferative and more quiescent iBECs states and there was no mean difference in TEER between early and late post-subculture time points. However, the expression of some TJP and AJP was dependent on length of subculture. Firstly, we found a significant loss of CLDN5 expression over time. Previous studies have also linked increased CLDN5 expression with a more angiogenic EC state [51]. Interestingly, CLDN5 overexpression promoted proliferation in hCMEC/D3 cells (immortalized human BEC line), while silencing it blocked cell cycle progression at the G0/G1 phase [52]. Although decreased CLDN5 levels are commonly associated with barrier disruption [53], upregulation of CLDN5 has also been seen in the context of diminished TEER and increased paracellular permeability $[11,54]$. Intense CLDN5 staining was observed at the junctional zones of more quiescent iBEC monolayers, supporting that, despite reduced expression, CLDN5 expression is sufficient, and it is appropriately localized to support the maintenance of high TEER. In agreement with existing literature, we found ZO-1 expression is significantly increased in more quiescent iBECs. Reduced expression of ZO-1 generally correlates with increased cell proliferation, such as in highly proliferative BECs from human brain tumors $[55,56]$. ZO- 1 has been reported to accumulate in the nucleus of proliferating epithelial cells [57], whereas in high density confluent cells, ZO-1 is primarily localized to cellcell junctions and inhibits cell proliferation by indirectly controlling expression of cell cycle regulators [57, 
58]. Our immunofluorescence analyses yielded clear evidence of enhanced ZO-1 junctional localization in more quiescent iBECs. We found OCLN levels did not change significantly as proliferation declined, indicating regulation of OCLN expression is likely not modulated by iBEC proliferative state.

For the AJPs evaluated, PECAM-1 expression was not significantly altered over time spent in subculture. However, we found a significant reduction in VE-cadherin expression. VE-cadherin is important in contact inhibition of proliferation [59]. Although its expression was decreased over time, VE-cadherin junctional localization was maintained at day 9 post-subculture, raising the possibility of its involvement in iBEC adoption of quiescence. Taken together, our data underscore that changes in the levels of any one TJP do not necessarily translate to changes in barrier tightness. This concept has been previously supported in studies demonstrating modulations of BBB permeability with no changes in protein levels [60-62]. How junctional remodeling in highly proliferative BECs occurs without loss of barrier integrity remains to be fully elucidated.

Prior studies have shown that iBECs are responsive to pro-inflammatory cytokine stimulation within two days post-subculture [63]. We explored the functional consequences of altered proliferation by comparing iBEC vulnerability to barrier disrupting insults on day 2 vs. day 9. TNF-a treatment produced comparable disruption in proliferative and more quiescent iBEC states. Therefore, the effects of TNF-a on TEER appear to be independent of the proliferative status of iBECs. Additionally, we modeled an Alzheimer's brain milieu using conditioned medium (CM) from neuronal cultures differentiated from hiPSCs harboring the Swedish mutation, a familial AD-causing mutation resulting in elevated $A \beta$ production [35]. We found that in proliferative iBECs, CM from hiPSC-neurons harboring the Swedish mutation (APPSwe/ ${ }^{\text {) }}$ ) caused a significant reduction in TEER, whereas CM from isogenic control hiPSC-neurons (APPWT) did not change TEER. In contrast, $\mathrm{CM}$ from neurons derived from either hiPSC line did not have a significant effect on TEER in more quiescent iBECs. These data suggest that BBB disruption in response to this AD-associated stimulus is dependent on iBEC proliferative state.

Our results align with a previous study showing a selective vulnerability of proliferative aortic ECs to the injurious effects of $A \beta[36]$ and suggest that actively dividing $B E C s$, such as those in injured or growth states, are more vulnerable to disruption by $A \beta$-associated metabolites. In vitro $A \beta$ treatment studies generally require concentrations in to $\mathrm{nM}$ to $\mu \mathrm{M}$ range to induce BBB disruption [64], whereas $A \beta-40$ and -42 levels in the APPSwe/+ $\mathrm{CM}$ were much lower, around $1000 \mathrm{pg} / \mathrm{mL}$ for $\mathrm{A} \beta-40$ and $100 \mathrm{pg} / \mathrm{mL}$ for $\mathrm{A} \beta-42$. It is possible that natural production by the hiPSC-derived neurons confers increased $A \beta$ toxicity, or that metabolites other than $A \beta$ produced as a result of the Swedish mutation are responsible for the barrier disrupting effects on iBECs. Interestingly, the presence of another familial AD-causing mutation in presenilin-1 or -2 in iBECs is not only associated with reduced barrier function, but also with impaired glycolysis [9]. As a future direction, iBEC metabolism could be evaluated after CM treatment to elucidate potential metabolic mechanisms driving the specific vulnerability of iBECs on day 2 post-subculture, providing insight into the relation between $B E C$ proliferation and BBB disruption in the context of $A D$. 
Both the barrier and the interface functions of the BBB are influenced by BEC proliferation. Maturation of the brain vasculature into the stable adult BBB depends on the establishment and maintenance of a predominantly quiescent BEC monolayer. On the other hand, aberrant brain angiogenesis caused by inappropriate BEC proliferation is observed in pathologies such as Alzheimer's disease (AD), glioblastoma multiforme, and cerebrovascular malformations [65-67]. A recent single-cell transcriptome analysis revealed that a subpopulation of angiogenic ECs is induced in the AD brain [68]. A central role for angiogenic vessels in the progression of $A D$ is suggested by their presence in brain regions affected by $A D$ pathology [65] and raises the possibility of inhibiting angiogenesis therapeutically [69]. A better understanding of the differential regulatory mechanisms of BBB functions in proliferating vs. quiescent BECs would inform novel therapeutic strategies to target vascular abnormalities and BEC activation in AD and other neurological diseases.

\section{Conclusion}

iBECs offer a model to study BBB-specific expressional and functional changes that occur as BEC proliferation declines.

\section{Abbreviations}

hiPSC: Human induced pluripotent stem cell; BEC: Brain endothelial cell; iBECs: hiPSC-derived brain endothelial-like cells; BBB: Blood-brain barrier; TEER: Transendothelial electrical resistance; BrdU: 5Bromo-2'-deoxyuridine (BrdU); TJP and AJP: Tight and adherens junction proteins; GLUT1: Glucose transporter-1; TNF-a: Tumor necrosis factor alpha; CM: Conditioned medium; AD: Alzheimer's disease; IFA: Immunofluorescence analyses; CLDN5: Claudin-5; VE-Cad: Vascular endothelial-cadherin; PECAM-1: Platelet endothelial cell adhesion molecule-1; ZO-1: Zona occludens-1 (ZO-1); OCLN: Occludin; HESFM: Human endothelial serum-free medium; bFGF: Basic fibroblast growth factor; RA: Retinoic acid; PBS: Phosphate buffered saline; NAD: Normal antibody diluent; MFI: Mean fluorescence intensity; ${ }^{14} \mathrm{C}-\mathrm{DG}:{ }^{14} \mathrm{C}$ 2-Deoxy-D-glucose; ${ }^{99 \mathrm{~m} T C-D T P A}$ : ${ }^{99 \mathrm{~m}}$ Tecnicium diethylenetriaminepentaacetic acid; APP: Amyloid precursor protein; Swe: Swedish mutation; ECs: Endothelial cells; NMC: No medium changes; SMC: Single medium change; CMC: Continual medium changes; PFKFB3: 6-Phosphofructo-2-kinase/fructose-2,6biphosphatase 3; HK2: Hexokinase 2; MCT1: Monocarboxylate transporter-1, Pe: Permeability-surface area coefficient; A: Amyloid beta; PTM: post-translational modification.

\section{Declarations}

\section{Ethics approval and consent to participate}

Not applicable.

\section{Consent for publication}

Not applicable. 
Availability of data and materials

Data are available from the corresponding author with reasonable request.

\section{Competing interests}

The authors declare no competing interests.

\section{Funding information}

This work is supported by grants from the National Institutes of Health-National Institute on Aging (AG065928, T32AG066574) and the US Department of Veterans Affairs.

\section{Authors' contributions}

Conception and design: LW, SE. Acquisition of the data: LW, TF, RW, KE. Analysis and interpretation of the data: $L W, R W, A L, K E, J Y, W B, S E$. Drafting the manuscript: $L W$. Critically revising the manuscript: $L W, A L$, $\mathrm{KE}, \mathrm{JY}, \mathrm{WB}, \mathrm{SE}$. All authors read and approved the final manuscript.

\section{Acknowledgements}

We wish to thank Dr. Ethan Lippmann for his generosity in sharing research protocols and his invaluable advice on model development.

\section{Author details}

${ }^{1}$ Veterans Affairs Puget Sound Health Care System, Geriatrics Research Education and Clinical Center (GRECC), Seattle, WA, USA. ${ }^{2}$ Nagasaki University Graduate School of Biomedical Sciences, Department of Neurosurgery, Nagasaki, Nagasaki, Japan. ${ }^{3}$ University of Washington School of Medicine, Department of Medicine, Division of Gerontology and Geriatric Medicine, Seattle, WA, USA. ${ }^{4}$ University of Washington, Department of Bioengineering, Seattle, WA, USA. ${ }^{5}$ University of Washington, Institute for Stem Cell and Regenerative Medicine, Seattle, WA, USA. ${ }^{6}$ University of Washington, Department of Laboratory Medicine and Pathology, Seattle, WA, USA.

\section{References}

1. Sweeney MD, Zhao Z, Montagne A, Nelson AR, Zlokovic BV. Blood-Brain Barrier: From Physiology to Disease and Back. Physiol Rev. 2019;99(1):21-78.

2. Liebner S, Dijkhuizen RM, Reiss Y, Plate KH, Agalliu D, Constantin G. Functional morphology of the blood-brain barrier in health and disease. Acta Neuropathol. 2018;135(3):311-36.

3. Erickson MA, Wilson ML, Banks WA. In vitro modeling of blood-brain barrier and interface functions in neuroimmune communication. Fluids Barriers CNS. 2020;17(1):26. 
4. Ben-Zvi A, Lacoste B, Kur E, Andreone BJ, Mayshar Y, Yan H, et al. Mfsd2a is critical for the formation and function of the blood-brain barrier. Nature. 2014;509(7501):507-11.

5. Erickson MA, Banks WA. Neuroimmune Axes of the Blood-Brain Barriers and Blood-Brain Interfaces: Bases for Physiological Regulation, Disease States, and Pharmacological Interventions. Pharmacol Rev. 2018;70(2):278-314.

6. Appelt-Menzel A, Cubukova A, Günther K, Edenhofer F, Piontek J, Krause G, et al. Establishment of a Human Blood-Brain Barrier Co-culture Model Mimicking the Neurovascular Unit Using Induced Pluriand Multipotent Stem Cells. Stem Cell Reports. 2017;8(4):894-906.

7. Lim RG, Quan C, Reyes-Ortiz AM, Lutz SE, Kedaigle AJ, Gipson TA, et al. Huntington's Disease iPSCDerived Brain Microvascular Endothelial Cells Reveal WNT-Mediated Angiogenic and Blood-Brain Barrier Deficits. Cell Rep. 2017;19(7):1365-77.

8. Vatine GD, Al-Ahmad A, Barriga BK, Svendsen S, Salim A, Garcia L, et al. Modeling Psychomotor Retardation using iPSCs from MCT8-Deficient Patients Indicates a Prominent Role for the BloodBrain Barrier. Cell Stem Cell. 2017;20(6):831-43.

9. Raut S, Patel R, Al-Ahmad AJ. Presence of a mutation in PSEN1 or PSEN2 gene is associated with an impaired brain endothelial cell phenotype in vitro. Fluids Barriers CNS. 2021;18(1):3.

10. Katt ME, Mayo LN, Ellis SE, Mahairaki V, Rothstein JD, Cheng L, et al. The role of mutations associated with familial neurodegenerative disorders on blood-brain barrier function in an iPSC model. Fluids Barriers CNS. 2019;16(1):20.

11. Oikari LE, Pandit R, Stewart R, Cuní-López C, Quek H, Sutharsan R, et al. Altered Brain Endothelial Cell Phenotype from a Familial Alzheimer Mutation and Its Potential Implications for Amyloid Clearance and Drug Delivery. Stem Cell Reports. 2020;14(5):924-39.

12. Lippmann ES, Azarin SM, Kay JE, Nessler RA, Wilson HK, Al-Ahmad A, et al. Derivation of blood-brain barrier endothelial cells from human pluripotent stem cells. Nat Biotechnol. 2012;30(8):783-91.

13. Lippmann ES, Al-Ahmad A, Azarin SM, Palecek SP, Shusta EV. A retinoic acid-enhanced, multicellular human blood-brain barrier model derived from stem cell sources. Sci Rep. 2014;4:4160.

14. Hollmann EK, Bailey AK, Potharazu AV, Neely MD, Bowman AB, Lippmann ES. Accelerated differentiation of human induced pluripotent stem cells to blood-brain barrier endothelial cells. Fluids Barriers CNS. 2017;14(1):9.

15. Neal EH, Marinelli NA, Shi Y, McClatchey PM, Balotin KM, Gullett DR, et al. A Simplified, Fully Defined Differentiation Scheme for Producing Blood-Brain Barrier Endothelial Cells from Human iPSCs. Stem Cell Reports. 2019;12(6):1380-8.

16. Winkler EA, Nishida Y, Sagare AP, Rege SV, Bell RD, Perlmutter D, et al. GLUT1 reductions exacerbate Alzheimer's disease vasculo-neuronal dysfunction and degeneration. Nat Neurosci. 2015;18(4):52130.

17. Koepsell H. Glucose transporters in brain in health and disease. Pflugers Arch. 2020;472(9):1299343. 
18. Gejl M, Brock B, Egefjord L, Vang K, Rungby J. and Gjedde A. Blood-Brain Glucose Transfer in Alzheimer's disease: Effect of GLP-1 Analog Treatment. Sci Rep. 2017;7(1):17490.

19. Westin JE, Lindgren HS, Gardi J, Nyengaard JR, Brundin P, Mohapel P, et al. Endothelial proliferation and increased blood-brain barrier permeability in the basal ganglia in a rat model of 3,4dihydroxyphenyl-L-alanine-induced dyskinesia. J Neurosci Res. 2006;26(37):9448-61.

20. Halder SK, Milner R. Mild hypoxia triggers transient blood-brain barrier disruption: a fundamental protective role for microglia. Acta Neuropathol Commun. 2020;8(1):175.

21. Zhu H, Zhang Y, Zhong Y, Ye Y, Hu X, Gu L, et al. Inflammation-Mediated Angiogenesis in Ischemic Stroke. Front Cell Neurosci. 2021;15:652647.

22. Steinman J, Sun HS, Feng ZP. Microvascular Alterations in Alzheimer's Disease. Front Cell Neurosci. 2021;14:618986.

23. Pajak B, Siwiak E, Sołtyka M, Priebe A, Zieliński R, Fokt I, et al. 2-Deoxy-d-Glucose and Its Analogs: From Diagnostic to Therapeutic Agents. Int J Mol Sci. 2019;21(1):234.

24. Dehouck MP, Méresse S, Delorme P, Fruchart JC, Cecchelli R. An easier, reproducible, and massproduction method to study the blood-brain barrier in vitro. J Neurochem. 1990;54(5):1798-801.

25. Young JE, Fong LK, Frankowski H, Petsko GA, Small SA, Goldstein LSB. Stabilizing the Retromer Complex in a Human Stem Cell Model of Alzheimer's Disease Reduces TAU Phosphorylation Independently of Amyloid Precursor Protein. Stem Cell Reports. 2018;10(3):1046-58.

26. Knupp A, Mishra S, Martinez R, Braggin JE, Szabo M, Kinoshita C, et al. Depletion of the AD Risk Gene SORL1 Selectively Impairs Neuronal Endosomal Traffic Independent of Amyloidogenic APP Processing. Cell Rep. 2020;31(9):107719.

27. D'Souza GX, Rose SE, Knupp A, Nicholson DA, Keene CD, Young JE. The application of in vitroderived human neurons in neurodegenerative disease modeling. J Neurosci Res. 2021;99(1):124-40.

28. Ricard N, Bailly S, Guignabert C, Simons M. The quiescent endothelium: signalling pathways regulating organ-specific endothelial normalcy. Nat Rev Cardiol. 2021;18(8):565-80.

29. Veys K, Fan Z, Ghobrial M, Bouché A, García-Caballero M, Conchinha NV, Vriens K, et al. Role of the GLUT1 Glucose Transporter in Postnatal CNS Angiogenesis and Blood-Brain Barrier Integrity. Circ Res. 2020;127(4):466-82.

30. Eelen G, de Zeeuw P, Treps L, Harjes U, Wong BW, Carmeliet P. Endothelial Cell Metabolism Physiol Rev. 2018;98(1):3-58.

31. De Bock K, Georgiadou M, Schoors S, Kuchnio A, Wong BW, Cantelmo AR, et al. Role of PFKFB3driven glycolysis in vessel sprouting. Cell. 2013;154(3):651-63.

32. DeWaal D, Nogueira V, Terry AR, Patra KC, Jeon SM, Guzman G, et al. Hexokinase-2 depletion inhibits glycolysis and induces oxidative phosphorylation in hepatocellular carcinoma and sensitizes to metformin. Nat Commun. 2018;9(1):446.

33. Pérez-Escuredo J, Van Hée VF, Sboarina M, Falces J, Payen VL, Pellerin L, et al. Monocarboxylate transporters in the brain and in cancer. Biochim Biophys Acta. 2016;1863(10):2481-97. 
34. Aghaee F, Pirayesh Islamian J, Baradaran B. Enhanced radiosensitivity and chemosensitivity of breast cancer cells by 2-deoxy-d-glucose in combination therapy. J Breast Cancer. 2012;15(2):141-7.

35. Sisodia SS, St George-Hyslop PH. gamma-Secretase, Notch, Abeta and Alzheimer's disease: where do the presenilins fit in? Nat Rev Neurosci. 2002;3(4):281-90.

36. Balcells M, Wallins JS, Edelman ER. Amyloid beta toxicity dependent upon endothelial cell state. Neurosci Lett. 2008;441(3):319-22.

37. Sun P. Contact inhibition against senescence. Oncotarget. 2014;5(17):7212-3.

38. Dumas SJ, García-Caballero M, Carmeliet P. Metabolic Signatures of Distinct Endothelial Phenotypes. Trends Endocrinol Metab. 2020;31(8):580-95.

39. Kumagai AK, Kang YS, Boado RJ, Pardridge WM. Upregulation of blood-brain barrier GLUT1 glucose transporter protein and mRNA in experimental chronic hypoglycemia. Diabetes. 1995;44(12):1399404.

40. Yu P, Wilhelm K, Dubrac A, Tung JK, Alves TC, Fang JS, et al. FGF-dependent metabolic control of vascular development. Nature. 2017;545(7653):224-8.

41. Payen VL, Mina E, Van Hée VF, Porporato PE, Sonveaux P. Monocarboxylate transporters in cancer. Mol Metab. 2020;33:48-66.

42. Miranda-Gonçalves V, Bezerra F, Costa-Almeida R, Freitas-Cunha M, Soares R, Martinho O, et al. Monocarboxylate transporter 1 is a key player in glioma-endothelial cell crosstalk. Mol Carcinog. 2017;56(12):2630-42s.

43. Rebelos E, Rinne JO, Nuutila P, Ekblad LL. Brain Glucose Metabolism in Health, Obesity, and Cognitive Decline-Does Insulin Have Anything to Do with It? A Narrative Review. J Clin Med. 2021;10(7):1532.

44. Sweeney MD, Montagne A, Sagare AP, Nation DA, Schneider LS, Chui HC, et al. Vascular dysfunctionThe disregarded partner of Alzheimer's disease. Alzheimers Dement. 2019;15(1):158-67.

45. Akman Cl, Provenzano F, Wang D, Engelstad K, Hinton V, Yu J, et al. Topography of brain glucose hypometabolism and epileptic network in glucose transporter 1 deficiency. Epilepsy Res. 2015;110:206-15.

46. Sone H, Deo BK, Kumagai AK. Enhancement of glucose transport by vascular endothelial growth factor in retinal endothelial cells. Invest Ophthalmol Vis Sci. 2000;41(7):1876-84.

47. Patching SG. Glucose Transporters at the Blood-Brain Barrier: Function, Regulation and Gateways for Drug Delivery. Mol Neurobiol. 2017;54(2):1046-77.

48. Jurcovicova J. Glucose transport in brain - effect of inflammation. Endocr Regul. 2014;48(1):35-48.

49. Yan Q, Lu Y, Zhou L, Chen J, Xu H, Cai M, et al. Mechanistic insights into GLUT1 activation and clustering revealed by super-resolution imaging. Proc Natl Acad Sci U S A. 2018;115(27):7033-8.

50. Lee EE, Ma J, Sacharidou A, Mi W, Salato VK, Nguyen N, et al. A Protein Kinase C Phosphorylation Motif in GLUT1 Affects Glucose Transport and is Mutated in GLUT1 Deficiency Syndrome. Mol Cell. 2015;58(5):845-53. 
51. Krishnan S, Szabo E, Burghardt I, Frei K, Tabatabai G, Weller M. Modulation of cerebral endothelial cell function by TGF- $\beta$ in glioblastoma: VEGF-dependent angiogenesis versus endothelial mesenchymal transition. Oncotarget. 2015;6(26):22480-95.

52. Ma SC, Li Q, Peng JY, Zhouwen JL, Diao JF, Niu JX, et al. Claudin-5 regulates blood-brain barrier permeability by modifying brain microvascular endothelial cell proliferation, migration, and adhesion to prevent lung cancer metastasis. CNS Neurosci Ther. 2017;23(12):947-60.

53. Nitta T, Hata M, Gotoh S, Seo Y, Sasaki H, Hashimoto N, et al. Size-selective loosening of the bloodbrain barrier in claudin-5-deficient mice. J Cell Biol. 2003;161(3):653-60.

54. Soma T, Chiba H, Kato-Mori Y, Wada T, Yamashita T, Kojima T, et al. $\operatorname{Thr}(207)$ of claudin-5 is involved in size-selective loosening of the endothelial barrier by cyclic AMP. Exp Cell Res. 2004;300(1):20212.

55. Bauer HC, Krizbai IA, Bauer H, Traweger A. "You Shall Not Pass"-tight junctions of the blood brain barrier. Front Neurosci. 2014;8:392.

56. Unger RE, Oltrogge JB, von Briesen $\mathrm{H}$, Engelhardt B, Woelki U, Schlote W, et al. Isolation and molecular characterization of brain microvascular endothelial cells from human brain tumors. In Vitro Cell Dev Biol Anim. 2002;38(5):273-81.

57. Gottardi CJ, Arpin M, Fanning AS, Louvard D. The junction-associated protein, zonula occludens-1, localizes to the nucleus before the maturation and during the remodeling of cell-cell contacts. Proc Natl Acad Sci U S A. 1996;93(20):10779-84.

58. Sourisseau T, Georgiadis A, Tsapara A, Ali RR, Pestell R, Matter K, et al. Regulation of PCNA and cyclin D1 expression and epithelial morphogenesis by the ZO-1-regulated transcription factor ZONAB/DbpA. Mol Cell Biol. 2006;26(6):2387-98.

59. Baumeister U, Funke R, Ebnet K, Vorschmitt H, Koch S, Vestweber D. Association of Csk to VEcadherin and inhibition of cell proliferation. EMBO J. 2005;24(9):1686-95.

60. Stamatovic SM, Keep RF, Wang MM, Jankovic I, Andjelkovic AV. Caveolae-mediated internalization of occludin and claudin-5 during CCL2-induced tight junction remodeling in brain endothelial cells. $J$ Biol Chem. 2009;284(28):19053-66.

61. Hamm S, Dehouck B, Kraus J, Wolburg-Buchholz K, Wolburg H, Risau W, et al. Astrocyte mediated modulation of blood-brain barrier permeability does not correlate with a loss of tight junction proteins from the cellular contacts. Cell Tissue Res. 2004;315(2):157-66.

62. Rubin LL, Staddon JM. The cell biology of the blood-brain barrier. Annu Rev Neurosci. 1999;22:1128.

63. Mantle JL, Lee KH. A differentiating neural stem cell-derived astrocytic population mitigates the inflammatory effects of TNF- $a$ and IL- 6 in an iPSC-based blood-brain barrier model. Neurobiol Dis. 2018;119:113-20.

64. Qosa H, LeVine H 3rd, Keller JN, Kaddoumi A. Mixed oligomers and monomeric amyloid- $\beta$ disrupts endothelial cells integrity and reduces monomeric amyloid- $\beta$ transport across $\mathrm{hCMEC/D} 3$ cell line as an in vitro blood-brain barrier model. Biochim Biophys Acta. 2014;1842(9):1806-15. 
65. Desai BS, Schneider JA, Li JL, Carvey PM, Hendey B. Evidence of angiogenic vessels in Alzheimer's disease. J Neural Transm (Vienna). 2009;116(5):587-97.

66. Giusti I, Delle Monache S, Di Francesco M, Sanità P, D'Ascenzo S, Gravina GL, et al. From glioblastoma to endothelial cells through extracellular vesicles: messages for angiogenesis. Tumour Biol. 2016;37(9):12743-53.

67. Sure U, Butz N, Schlegel J, Siegel AM, Wakat JP, Mennel HD, et al. Endothelial proliferation, neoangiogenesis, and potential de novo generation of cerebrovascular malformations. J Neurosurg. 2001;94(6):972-7.

68. Lau SF, Cao H, Fu AKY, Ip NY. Single-nucleus transcriptome analysis reveals dysregulation of angiogenic endothelial cells and neuroprotective glia in Alzheimer's disease. Proc Natl Acad Sci U S A. 2020;117(41):25800-9.

69. Vagnucci AH Jr, Li WW. Alzheimer's disease and angiogenesis. Lancet. 2003;361(9357):605-8.

\section{Table}

Table 1: Antibodies 


\begin{tabular}{|c|c|c|c|}
\hline Target antigen & Vendor & Catalog Number & Dilution \\
\hline 5-bromo-2'-deoxyuridine (BrdU) & abcam & ab6326 & $1: 250$ \\
\hline PFKFB3 & Cell Signaling & $13123 S$ & $1: 500$ \\
\hline GLUT1 & EMD Millipore & 07-1401 & $1: 10000$ \\
\hline HK2 & Cell Signaling & $2867 \mathrm{~T}$ & $1: 1000$ \\
\hline MCT1 & Proteintech & 20139-1-AP & $1: 10000$ \\
\hline CLDN5 & ThermoFisher Scientific & $35-2500$ & $1: 50$ \\
\hline OCLN & ThermoFisher Scientific & $33-1500$ & $1: 50$ \\
\hline ZO-1 & ThermoFisher Scientific & $61-7300$ & $1: 25$ \\
\hline PECAM-1 & Sigma & P8590 & $1: 25$ \\
\hline VE-Cad & R\&D Systems & AF938 & $1: 25$ \\
\hline -actin & Cell Signaling & $3700 s$ & $1: 10000$ \\
\hline -actin, HRP-linked & abcam & ab49900 & $1: 20000$ \\
\hline Anti-mouse lgG, HRP-linked & Jackson ImmunoResearch & $115-035-003$ & $1: 5000$ \\
\hline Anti-rabbit IgG, HRP-linked & Jackson ImmunoResearch & $111-035-144$ & $1: 5000$ \\
\hline AlexaFluor® 488- & ThermoFisher Scientific & A-21208 & $1: 500$ \\
\hline \multicolumn{4}{|l|}{ Anti-rat IgG } \\
\hline AlexaFluor ${ }^{\circledR} 488$ - & Jackson ImmunoResearch & $715-545-150$ & $1: 200$ \\
\hline \multicolumn{4}{|l|}{ Anti-mouse IgG } \\
\hline AlexaFluor® 594- & Jackson ImmunoResearch & $711-585-152$ & $1: 200$ \\
\hline \multicolumn{4}{|l|}{ Anti-rabbit lgG } \\
\hline AlexaFluor® 594- & Jackson ImmunoResearch & $705-585-147$ & $1: 200$ \\
\hline Anti-goat lgG & & & \\
\hline
\end{tabular}

\section{Figures}


a

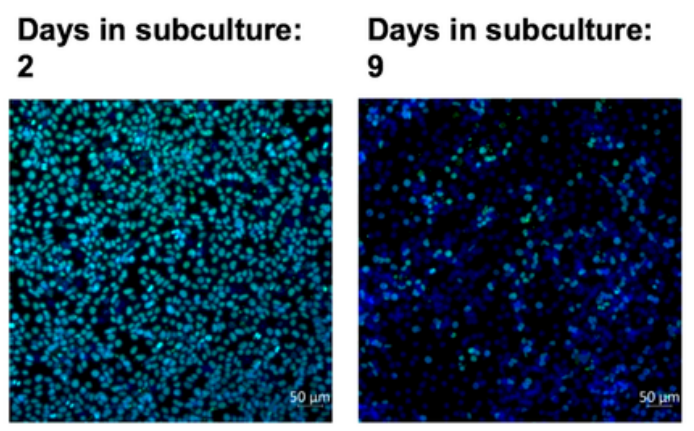

b
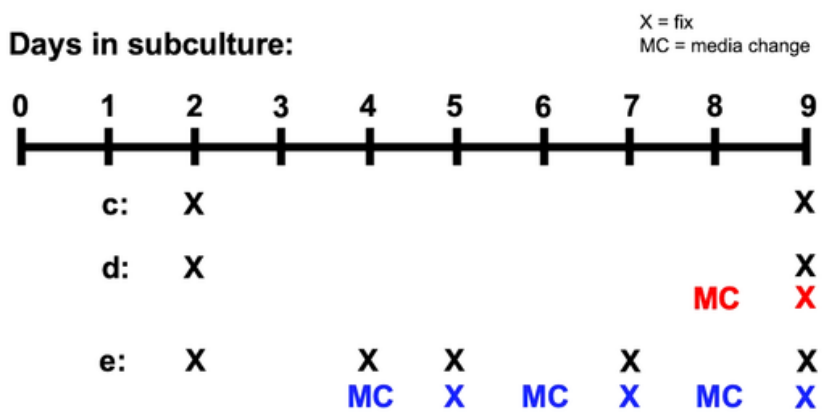

d

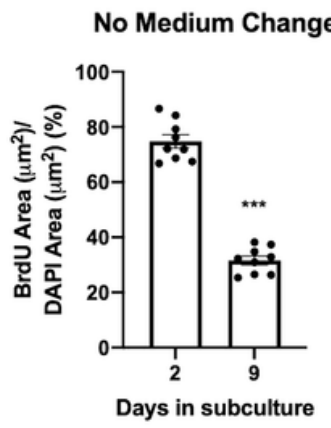

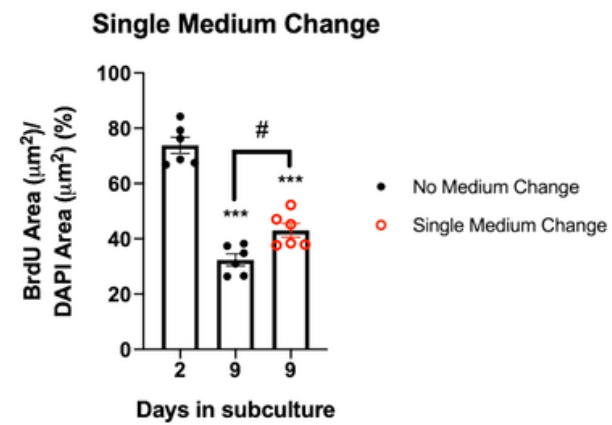

e

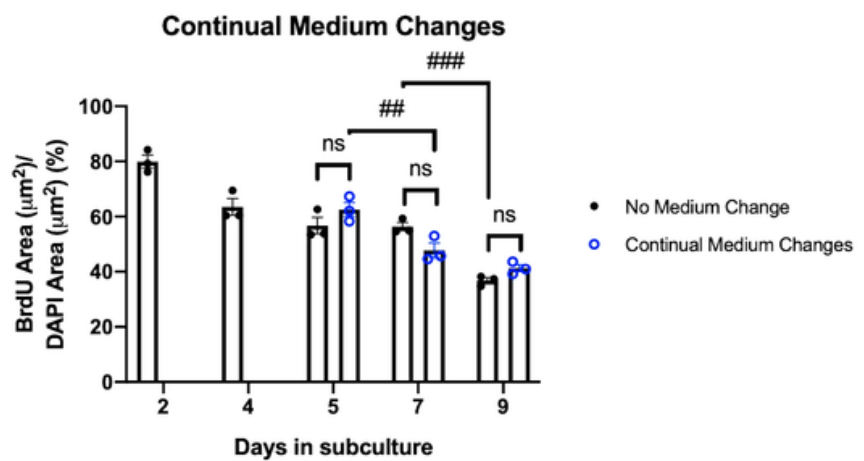

Figure 1

Effects of medium changes on BrdU incorporation in GM25256 iBECs. a. Representative immunocytochemistry of BrdU (green) incorporation in iBECs on days 2 and 9 after subculture, counterstained with DAPI (blue). b. Schematic depicting days of iBEC fixation (X) or media change (MC) corresponding to graphs c-e. c. Immunofluorescence analysis of BrdU+ area/DAPI+ area in iBECs with no MC on days 2 and 9 after subculture. Each data point represents the average of 3-4 random fields of view per well. Three independent differentiations were performed with $n=3$ wells imaged per group per differentiation. ${ }^{* \star}$ p $<0.001$ (Unpaired two-tailed t-test). d. Immunofluorescence analysis of BrdU+ area/DAPI+ area in iBECs days 2 and 9 after subculture with no MC vs. on day 9 with a single MC on day 8. Each data point represents the average of 3-4 random fields of view per well. Two independent differentiations were performed with $n=3$ wells imaged per group per differentiation. $\# p<0.05,{ }^{\star * *} p<0.001$ (vs. day 2) (One-way ANOVA with Tukey's multiple comparisons test). e. Immunofluorescence analysis of BrdU+ area/DAPI+ area in iBECs on days 2, 4, 5, 7 and 9 after subculture with no MC vs. on days 5, 7, and 9 with continual MC on days 4, 6, and 8 (day 5: single MC on day 4; day 7: two MC on days 4 and 6; day 9: three MC on days 4, 6, and 8. Each data point represents the average of 3 random fields of view per well. Not significant (ns), \#\#p<0.01, \#\#\#p<0.001 (Two-way ANOVA with Tukey's multiple comparisons test used to compare no media change vs. continual media change groups on days 5,7 , and 9 ). c-e. Means are displayed with their SE and $n$. 
a
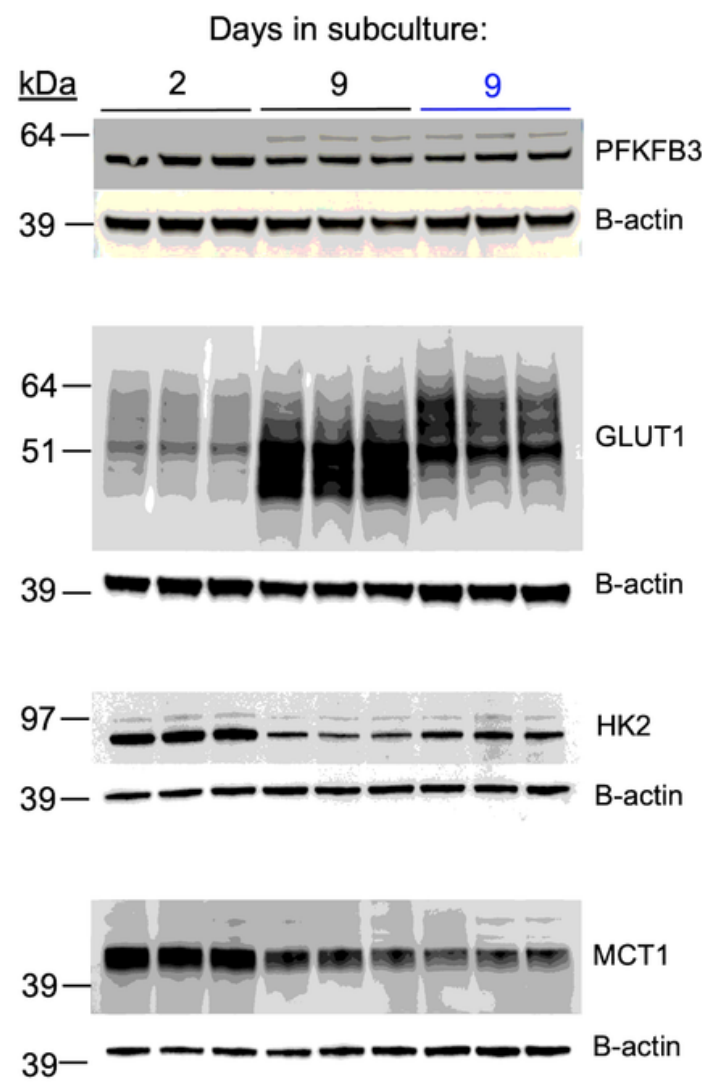

b

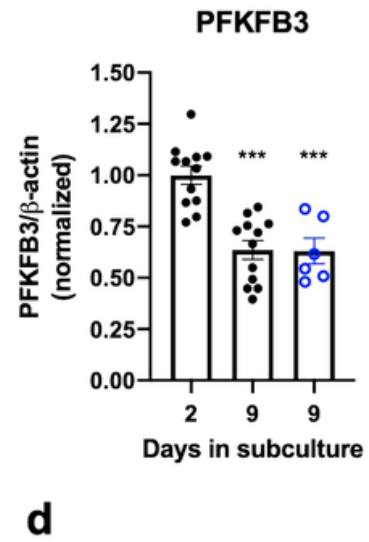

HK2

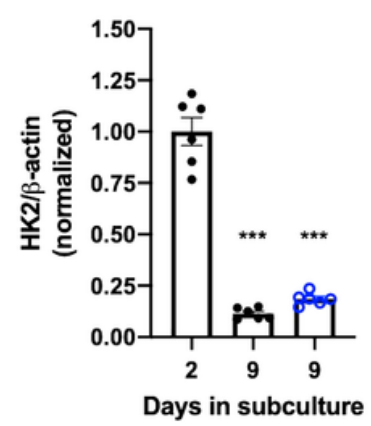

C

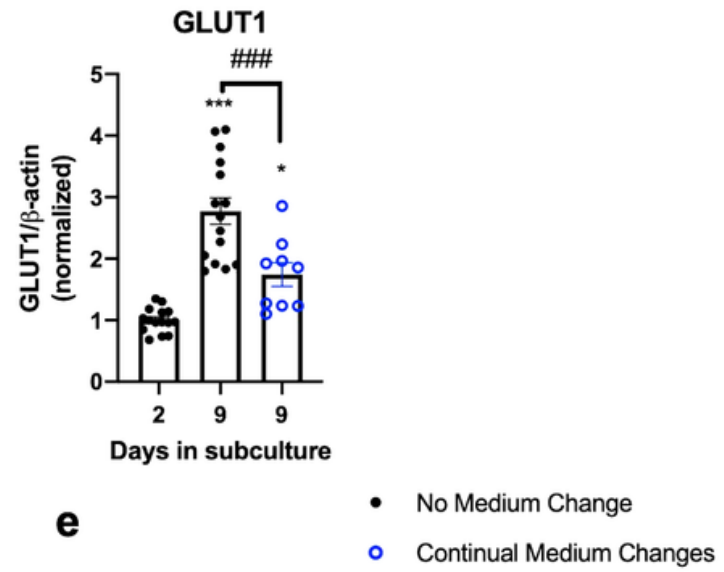

MCT1

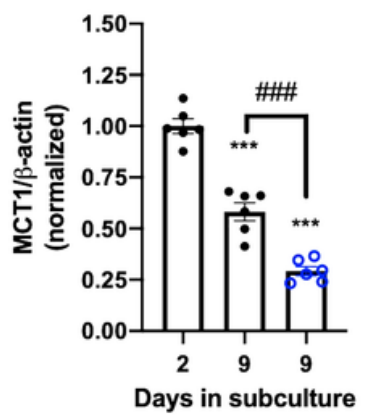

Figure 2

Effects of length of subculture and medium changes (MC) on iBEC expression of glucose metabolism/transport-related proteins. a. Representative immunoblots of PFKFB3, GLUT1, HK2, and MCT1 expression on days 2 and 9 after subculture with no MC (black) vs. on day 9 after continual MC on days 4, 6, and 8 (blue). b-e. Quantification of immunoblots. Expression was calculated relative to $\beta$-actin and normalized to day 2. Each data point represents one well. For PFKFB3 and GLUT1, two independent differentiations were performed with $n=3$ wells per group to evaluate expression of days 2 and 9 after subculture with no media changes. Then, two more independent differentiations were performed with $n=3$ wells per group to additionally evaluate expression on day 9 with continual media changes on days 4, 6 , and 8. For HK2 and MCT1, two independent differentiations were performed which evaluated all three groups with $n=3$ wells per group. ${ }^{*} p<0.05$, ${ }^{\star *} p<0.001$ (vs. day 2); \#\#\#p<0.001 (One-way ANOVA with Tukey's multiple comparisons test). Means are displayed with their SE and $\mathrm{n}$. 
a

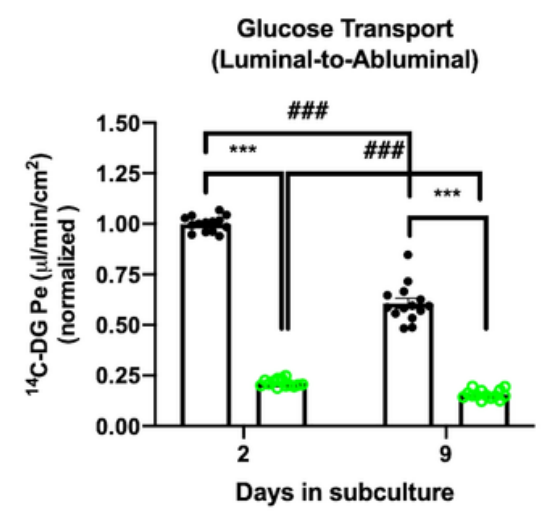

b

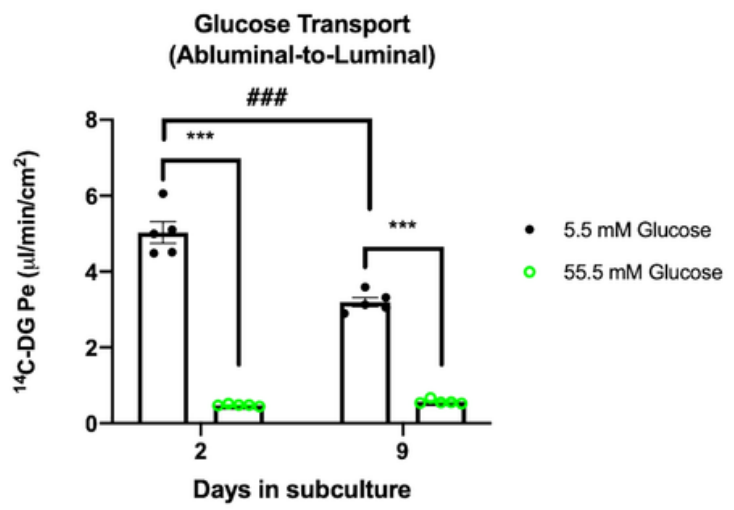

C

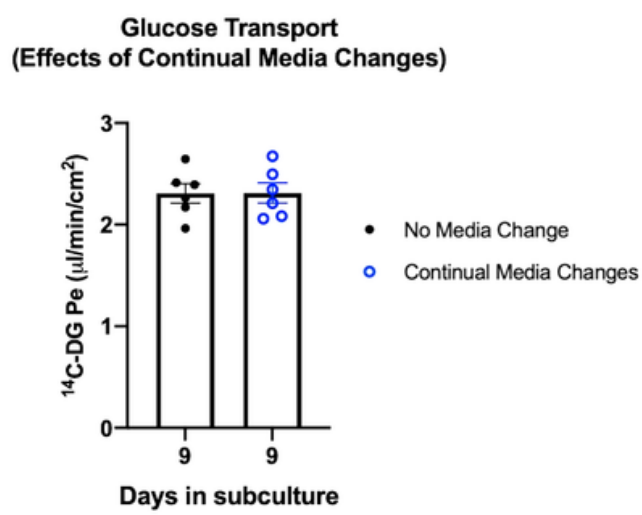

Figure 3

Glucose transport kinetics in GM25256 iBECs. a. Comparison of luminal-to-abluminal glucose transport saturability kinetics on days 2 and 9 after subculture. Comparison of abluminal-to-luminal glucose transport saturability kinetics on days 2 and 9 after subculture. Glucose transport rates (14C-DG Pe) were compared in the presence (55.5 mM Glucose, green) or absence (5.5 mM Glucose, black) of excess glucose in the luminal compartment to examine glucose transport system saturability. Three independent differentiations were performed to with $n=4-5$ transwells per group. 14C-DG Pe values were normalized to the unsaturated $\mathrm{Pe}$ on day 2. b. Comparison of abluminal-to-luminal glucose transport saturability kinetics on days 2 and 9 after subculture. 14C-DG Pe values were compared in the presence $(55.5 \mathrm{mM}$ Glucose, green) or absence ( $5.5 \mathrm{mM}$ Glucose, black) of excess glucose in the abluminal compartment to examine glucose transport system saturability. One differentiation was performed with $n=5$ transwells per group. $* \star \star \# \# \# p<0.001$ (Two-way ANOVA with Tukey's multiple comparisons test). b. Luminal-toabluminal 14C-DG Pe on day 9 after subculture were compared with (blue) and without (black) continual media changes on days 4,6 , and 8 . One differentiation was performed with $n=6$ transwells per group. (Unpaired two-tailed t-test). a, b. Means are displayed with their SE and n. 
a

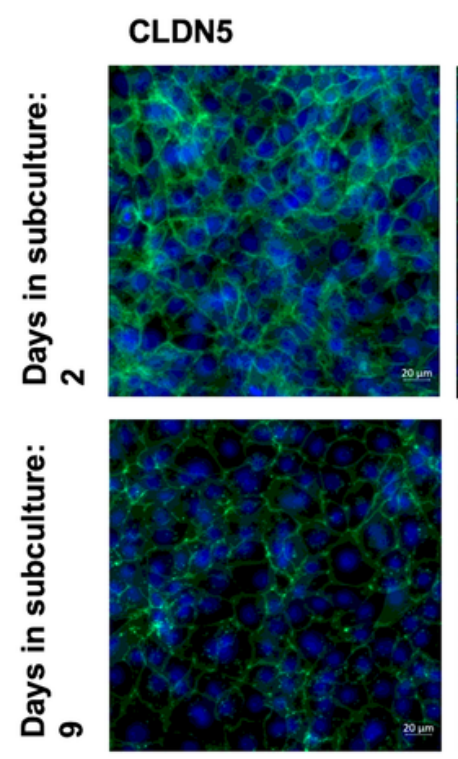

b

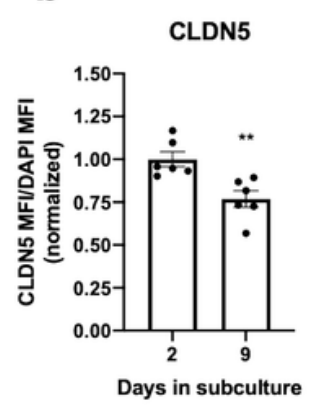

OCLN
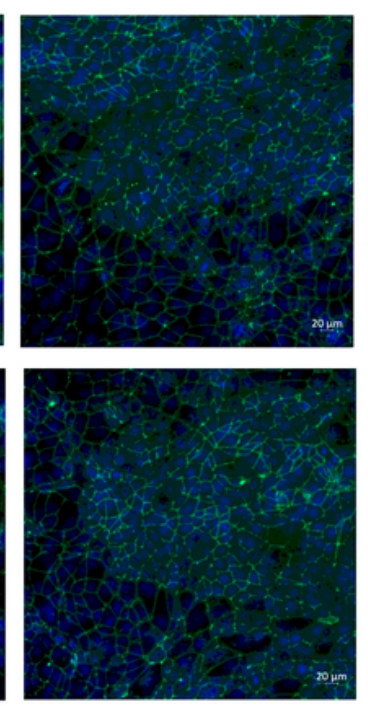

c

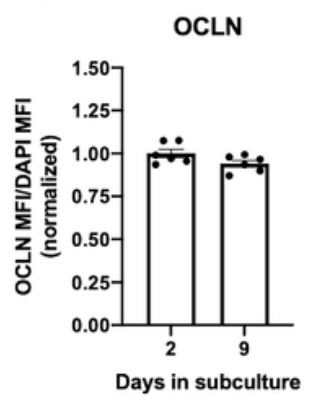

ZO-1
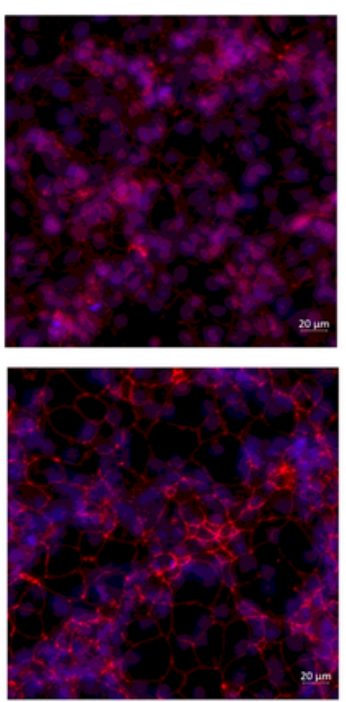

d

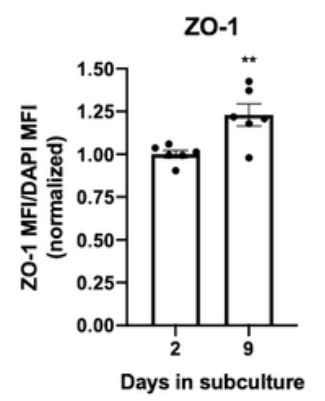

PECAM-1
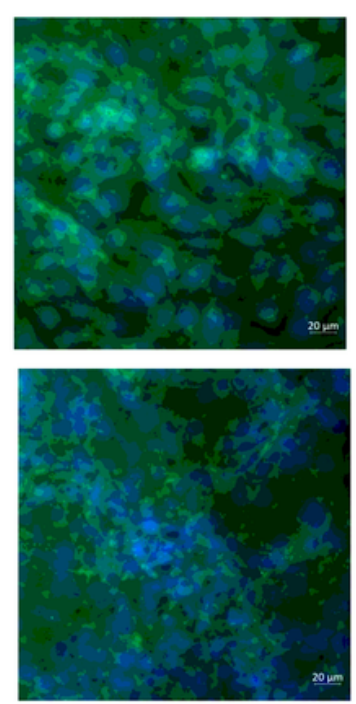

e

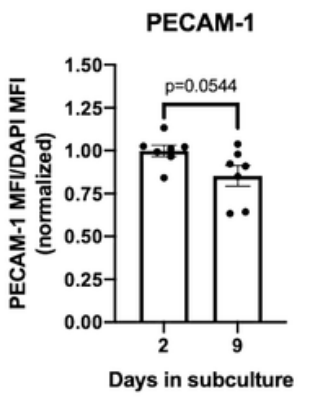

VE-Cad
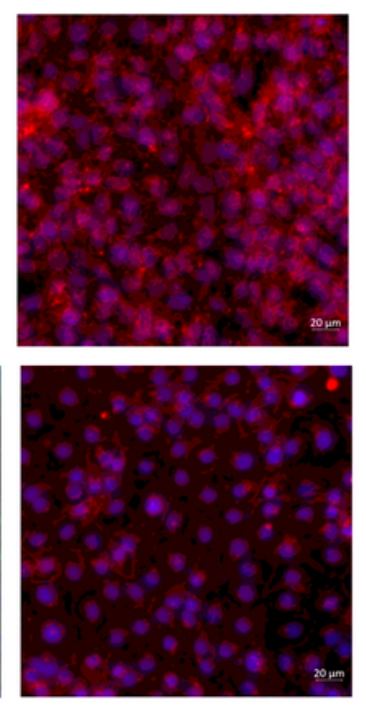

f

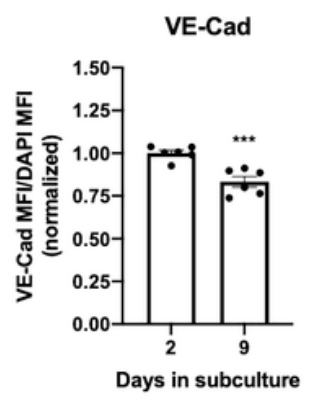

g

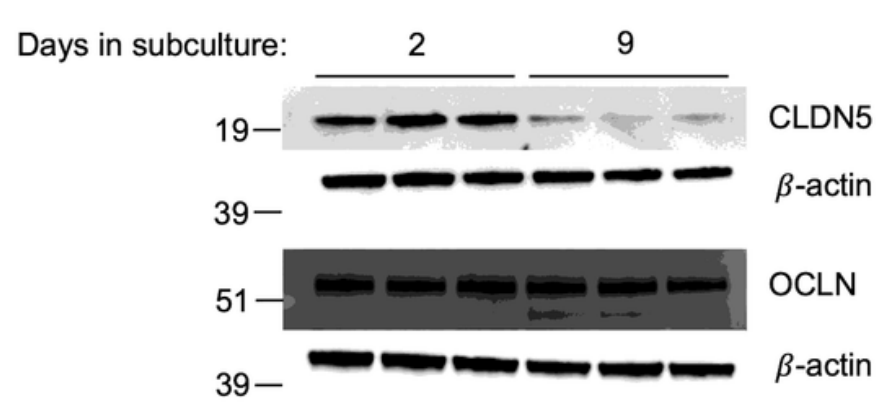

h

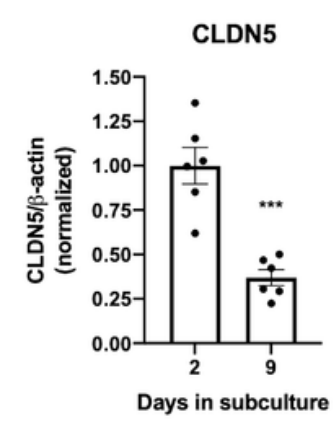

i

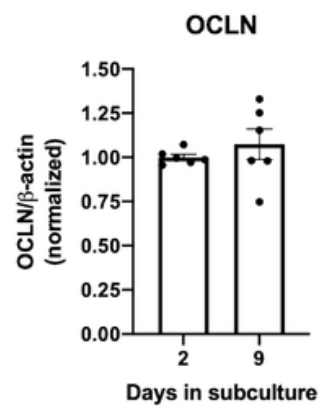

\section{Figure 4}

Immunocytochemistry and immunofluorescent analysis of TJP and AJP in GM25256 iBECs. a.

Representative immunocytochemistry of CLDN5 (green), OCLN (green), ZO-1 (red), PECAM-1 (green), and VE-Cad (red) on days 2 and 9 after subculture, counterstained with DAPI (blue). b-f. Immunofluorescence analysis of CLDN5, OCLN, ZO-1, PECAM-1, and VE-Cad mean fluorescence intensities (MFI) relative to DAPI MFI on days 2 and 9 after subculture (normalized to day 2). Each data point represents the average of 3-4 random fields of view per well. Two independent differentiations were performed with $n=3$ wells 
imaged per differentiation. ${ }^{* \star} p<0.01,{ }^{* \star *} p<0.001$ (Unpaired two-tailed t-test). g. Representative immunoblots of CLDN5 and OCLN expression on days 2 and 9 after subculture. h-i. Quantification of CLDN5 and OCLN immunoblots. Expression was calculated relative to $\beta$-actin and normalized to day 2 . Each data point represents one well. Two independent differentiations were performed with $n=3$ wells for each differentiation. ${ }^{* *} \mathrm{p}<0.001$ (Unpaired two-tailed t-test). b-f, h,i. Means are displayed with their SE and n.

a

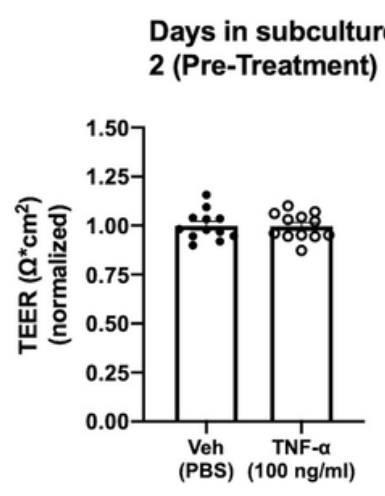

b

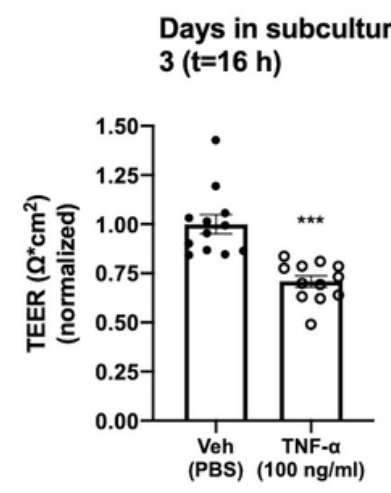

C

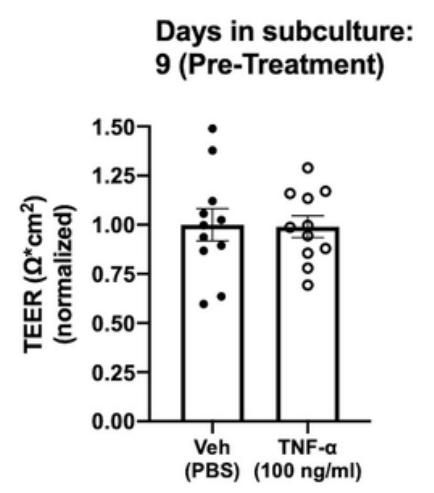

Williams et al., 2021. Figure 5

d

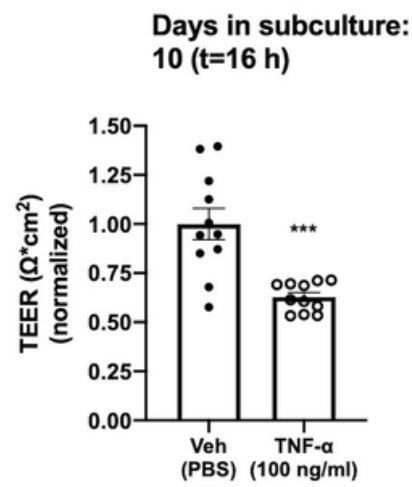

\section{Figure 5}

Effects of TNF- $\bigotimes$ treatment on TEER. a-d. On day 2 or day 9, transwells were organized into treatment groups such that TEER means were approximately equal and treated with $100 \mathrm{ng} / \mathrm{ml}$ TNF- $囚$ or PBS vehicle (Veh) control. TEER was measured after $16 \mathrm{~h}$ treatment, on day 3 or day 10 , respectively. TEER values were normalized to Veh control. Two independent differentiations of iBECs were performed with $n=5-7$ transwells per group. ${ }^{* *} \mathrm{p}<0.001$ (Unpaired two-tailed t-test). 
a

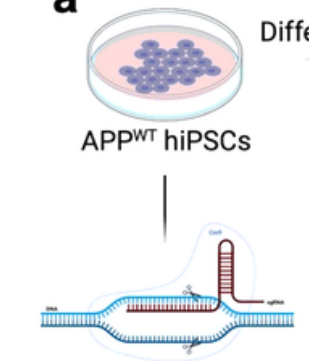

Insert Swedish mutation via CRISPR-Cas9

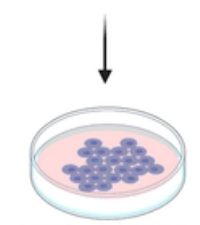

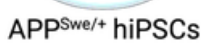

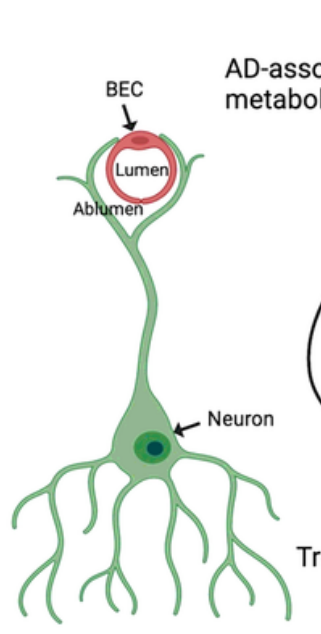

b

Days in subculture: 2 (Pre-Treatment)

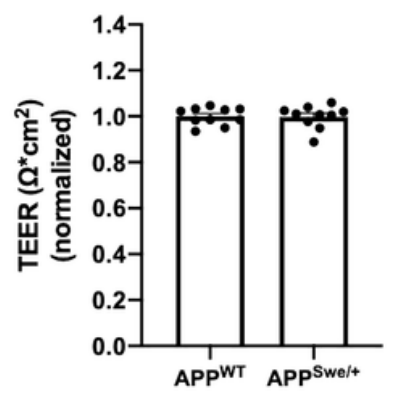

d

Days in subculture: 9 (Pre-Treatment)

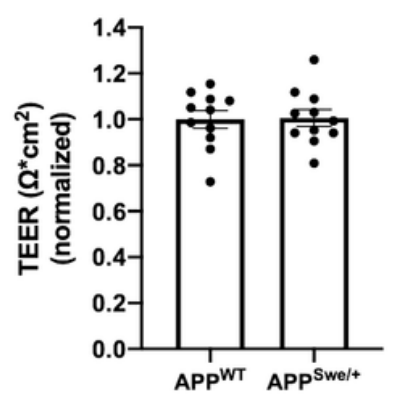

C

Days in subculture: 3 ( $t=24 \mathrm{~h}$ )

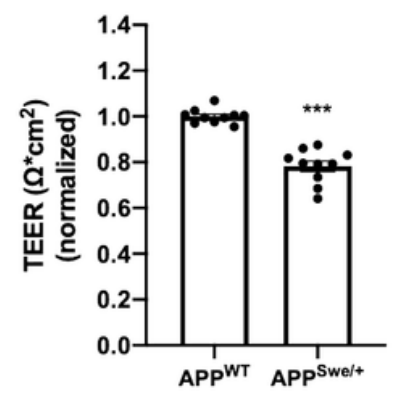

Days in subculture: $10(t=24 h)$

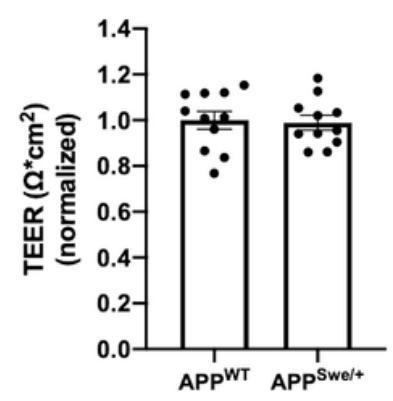

\section{Figure 6}

Modeling neuron-endothelial interactions in Alzheimer's disease using iBECs treated with conditioned media (CM) from hiPSC-derived neurons. a. Schematic depicting CM treatments. b-e. On day 2 or day 9, transwells were organized into treatment groups (APPWT and APPSwe/+) such that TEER means were approximately equal and treated with hiPSC-neuronal $\mathrm{CM}$. The $\mathrm{CM}$ effect on iBEC TEER was measured after $24 \mathrm{~h}$ treatment, on day 3 or day 10, respectively. TEER values were normalized to APPWT control. Two independent differentiations of iBECs were conducted to test conditioned media from one differentiation of hiPSC-neurons ( $n=3-4$ transwells per group). Results were confirmed with one additional differentiation of iBECs treated with CM from another differentiation of hiPSC-neurons ( $n=4-5$ transwells per group). ${ }^{* \star} \mathrm{p}<0.001$ (Unpaired two-tailed $\mathrm{t}$-test). Means are displayed with their SE and $\mathrm{n}$.

\section{Supplementary Files}

This is a list of supplementary files associated with this preprint. Click to download. 
- FigureS1.tiff

- Figures2.tiff

- Figures3.tiff

- Figures4.tiff

- FigureS5.tiff 